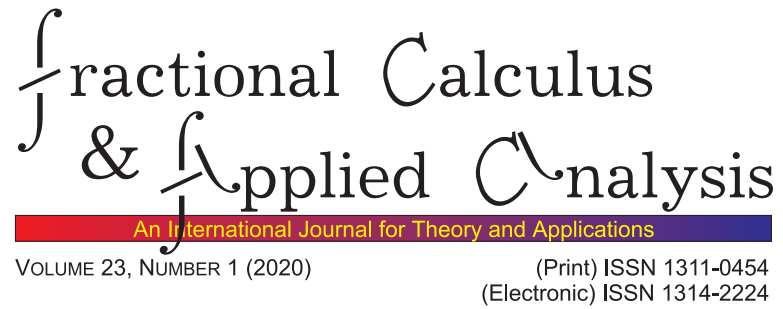

\title{
SURVEY PAPER
}

\section{CROSSOVER DYNAMICS FROM SUPERDIFFUSION TO SUBDIFFUSION: MODELS AND SOLUTIONS}

\author{
Emad Awad ${ }^{1}$, Ralf Metzler ${ }^{2}$
}

\begin{abstract}
The Cattaneo or telegrapher's equation describes the crossover from initial ballistic to normal diffusion. Here we study and survey time-fractional generalisations of this equation that are shown to produce the crossover of the mean squared displacement from superdiffusion to subdiffusion. Conditional solutions are derived in terms of Fox $H$-functions and the $\delta$ th-order moments as well as the diffusive flux of the different models are derived. Moreover, the concept of the distribution-like is proposed as an alternative to the probability density function.
\end{abstract}

MSC 2010: Primary 26A33, 33E12; Secondary 34A08, 35R11

Key Words and Phrases: Cattaneo equation; telegrapher's equation; crossover dynamics; fractional dynamic equations; anomalous diffusion; superdiffusion and subdiffusion; Fox $H$-functions

\section{Introduction}

Brownian diffusion processes [18, 81, 37] are characterised by the linear growth in time of the mean squared displacement $(\mathrm{MSD}),\left\langle x_{\varrho}^{2}(t)\right\rangle \sim t$. This process is governed by the coupled system of partial differential equations,

$$
J(x, t)=-\partial_{x} \varrho(x, t), \quad-\partial_{x} J(x, t)=\partial_{t} \varrho(x, t),
$$

here used in dimensionless units, respectively representing the constitutive equation (Fick's first law) and the continuity equation, where $J(x, t)$ is the diffusive flux and $\varrho(x, t)$ is the distribution of the diffusing substance at

(c) 2020 Diogenes Co., Sofia

pp. 55-102, DOI: $10.1515 /$ fca-2020-0003

DE GRUYTER 
position $x$ and time $t$. Combining both equations, we obtain the diffusion equation (Fick's second law) $\partial_{t} \varrho(x, t)=\partial_{x}^{2} \varrho(x, t)$. Here, $\partial_{\zeta}^{n}$ denotes the nth partial derivative with respect to the variable $\zeta$. Equations (1.1) subject to the initial condition $\varrho\left(x, 0^{+}\right)=\delta(x), x \in \mathbb{R}$, in terms of the Dirac delta function (unit impulse function), and the "natural" boundary conditions $\lim _{|x| \rightarrow \infty} \varrho(x, t)=0$, produce the famed Gaussian shape of $\varrho(x, t)$ with the linear growth of the MSD of the diffusing particles.

In this formulation Brownian motion is overdamped, i.e., any inertial effects of the diffusing tracer particle are considered to have decayed. In the original Langevin equation formulation, the particle motion at times shorter than the relaxation time (given in terms of the particle mobility) is inertia-dominated and thus ballistic [37, 34]. One way to incorporate the crossover from initial ballistic motion $\left\langle x^{2}(t)\right\rangle \sim t^{2}$ to final diffusive motion $\left\langle x^{2}(t)\right\rangle \sim t$ was achieved in the discussion of heat transport 33] in the form of the Cattaneo or telegrapher's equation discussed in Section 2 , Today, this field has adopted more general stochastic processes to account for the ever better resolved patterns involved in heat transport, [17. In this paper we study and survey the mathematical properties of generalised Cattaneo-type equations, in which the integer scaling exponents of ballistic and diffusive motion are replaced by given real-valued exponents.

Already in 1926 Richardson reported a process which deviates from equations (1.1) in the form of the cubic time dependence of the relative MSD of two particles in a turbulent flow [68]. In a wide variety of systems, the MSD of the diffusing particles follows the power-law form [6, 48, 49]

$$
\left\langle x_{\varrho}^{2}(t)\right\rangle \sim t^{\beta}, \beta>0,
$$

distinguishing subdiffusive processes with $0<\beta<1$, the normal Brownian process with $\beta=1$, superdiffusive processes with $1<\beta<2$, the ballistic process with $\beta=2$, and superballistic processes, $\beta>2$.

To describe such anomalous diffusion processes, fractional kinetic equations as proposed by Schneider and Wyss [88, 79], Zaslavsky 90, and Nigmatullin [61], are found to be outstanding tools. The time-fractional diffusion equation, for instance, given in the natural (Caputo) form [40, 41, 42]

$$
J_{C}(x, t)=-\partial_{x} \varrho(x, t), \quad-\partial_{x} J_{C}(x, t)={ }_{0^{+}}^{C} D_{t}^{\beta} \varrho(x, t),
$$

or in the modified (Riemann-Liouville) form

$$
J_{R L}(x, t)=-{ }_{0^{+}}^{R L} D_{t}^{1-\beta} \partial_{x} \varrho(x, t), \quad-\partial_{x} J_{R L}(x, t)=\partial_{t} \varrho(x, t),
$$

capture well the subdiffusive regime for $\varrho\left(x, 0^{+}\right)=\delta(x), x \in \mathbb{R}$, and $\lim _{|x| \rightarrow \infty} \varrho(x, t)=0$. Here ${ }_{0^{+}}^{C} D_{t}^{\beta}$ and ${ }_{0^{+}}^{R L} D_{t}^{\beta}, 0<\beta<1$, are respectively the Caputo and Riemann-Liouville fractional derivatives (see Appendix A). 
Similarly, the time-fractional wave equation in the natural form

$$
{ }_{0^{+}}^{C} D_{t}^{\beta} J_{C}(x, t)=-\partial_{x} \varrho(x, t), \quad-\partial_{x} J_{C}(x, t)={ }_{0^{+}}^{C} D_{t}^{\beta} \varrho(x, t),
$$

or in the modified form

$$
{ }_{0^{+}}^{R L} D_{t}^{\beta} J_{R L}(x, t)=-{ }_{0^{+}}^{R L} D_{t}^{1-\beta} \partial_{x} \varrho(x, t), \quad-\partial_{x} J_{R L}(x, t)=\partial_{t} \varrho(x, t),
$$

$0<\beta<1$, cover the superdiffusive regime when $\varrho\left(x, 0^{+}\right)=\delta(x), x \in \mathbb{R}$, and $\lim _{|x| \rightarrow \infty} \varrho(x, t)=0$.

Several early studies suggested that the scaling behaviour in terms of a single exponent, equation (1.2), is unable to describe many physical processes, such as the Sinai diffusion model [80, truncated Lévy-flights [44, or subdiffusive motion in velocity fields [50]. By now, the mono-scaling behaviour has been challenged to describe the temporal variation of the MSD in biological cells [4, 29, 63, 57, in protein surface water [84, phospholipids and cholesterols in a lipid bilayer [31, 32, 54], and in viscoelastic flows [89], see also the reviews [55, 47] and the references therein. These works were the basis for a string of papers [11, 12, 13, 14, 82, 15, 72] in which fractional order derivatives in the fractional diffusion equations (1.3) and (1.4) are replaced by derivatives of distributed-order [9]. Distributed-order time or space fractional diffusion equations are shown to be a versatile tool for the mathematical description of physical processes that become less anomalous in the course of time (accelerating subdiffusion and decelerating superdiffusion) or more anomalous in the course of time (retarding subdiffusion and accelerating superdiffusion).

In [16] a generalised Cattaneo-type equation was proposed, yielding the dynamic crossover behaviour

$$
\left\langle x_{\varrho}^{2}(t)\right\rangle \sim \begin{cases}t^{2 \alpha}, & t \rightarrow 0^{+} \\ t^{\alpha}, & t \rightarrow \infty\end{cases}
$$

where $0<\alpha<1$. When $0<\alpha<0.5$, equation (1.7) represents a retarded subdiffusion process, which can be considered a special case of the doubleorder time fractional diffusion equation of the natural type $\left(\beta_{2}=2 \beta_{1}\right)$ [11], and when $0.5<\alpha<1$, it represents a transition from the superdiffusive to subdiffusive regime, see also [65, 45, 2]. In the case $\alpha=1$ we recover the characteristic property of the classical Cattaneo model, see equation (2.2) below, the crossover from ballistic to normal diffusion. The reader is also referred to generalisations of diffusion, Fokker-Planck, and diffusion-wave equations by introduction of a generalised memory kernel [73, 74, 75]. However, recent numerical simulations have shown different transitions from superdiffusion to subdiffusion which are not, in all cases, covered by equation (1.7), see e.g., quasiperiodic interacting systems [38] and dewettingspreading-wetting transitions of self-propelled (run-and-tumble) clustered disks in a substrate with randomly placed pinning sites [76]. 
In this work, we present different forms of generalised Cattaneo-type equations, based on single-order and distributed-order time-fractional derivatives, which provide a variety of transitions from superdiffusive to subdiffusive motion. We first outline these forms in Section 2. Conditional solutions for the distributions of the proposed models are derived in terms of Fox $H$-functions, and the corresponding $\delta$ th-order moments are discussed in Section 3. In Section 4 we consider the diffusive flux, an important measurable quantity. Moreover, we introduce and study the so-called distributionlike, a quantity similar to the physical distribution, from which we obtain alternative formulations for the diffusion, fractional diffusion, and fractional Cattaneo-type diffusion equations. The subordination of time-fractional Cattaneo-type diffusion to the Gaussian process is also discussed. Finally, we summarise our results, and draw our conclusions in Section 5. We give a brief outline of the mathematical preliminaries in Appendix $\mathrm{A}$ and show the methods of deriving our solutions by solving four problems in Appendix B.

\section{Generalised Cattaneo equations}

Several representative models were introduced during the last seven decades in order to address the shortcomings of the Fourier-Fickian law in modelling the necessary finite propagation speed in heat and signal transport. One of the most prominent is the Cattaneo model, also known as Maxwell-Cattaneo or telegrapher's equation [10, 58], as well as the phonon scattering model [25], and the parabolic and hyperbolic two-step models [70, 71]. The monograph [86] contains a comprehensive historical background on these developments, see also [3].

The Cattaneo equation in dimensionless form reads [10]

$$
\left(1+\partial_{t}\right) J(x, t)=-\partial_{x} \varrho(x, t), \quad-\partial_{x} J(x, t)=\partial_{t} \varrho(x, t),
$$

which encodes the characteristic ballistic-diffusive crossover property of the MSD [65, 46, 87]

$$
\left\langle x_{\varrho}^{2}(t)\right\rangle \sim \begin{cases}t^{2}, & t \rightarrow 0^{+} \\ t, & t \rightarrow \infty\end{cases}
$$

with an exact solution for $\varrho(x, t)$ in terms of modified Bessel functions [30]. The first fractional Cattaneo equation was proposed by Nonnenmacher and Nonnenmacher 62]. The time-fractional Cattaneo equation (TFCE) has two familiar forms [16, 2]. The first form (natural type), uses the Caputo fractional derivative,

GCE-I: $\left(1+{ }_{0^{+}}^{C} D_{t}^{\alpha}\right) J_{C}(x, t)=-\partial_{x} \varrho(x, t),-\partial_{x} J_{C}(x, t)={ }_{0^{+}}^{C} D_{t}^{\beta} \varrho(x, t)$, 
with $\varrho\left(x, 0^{+}\right)=\delta(x), J_{C}\left(x, 0^{+}\right)=0, x \in \mathbb{R}, \lim _{|x| \rightarrow \infty} \varrho(x, t)=0$, and $\alpha, \beta \in(0,1]$. The second form of the time-fractional Cattaneo equation uses the Riemann-Liouville fractional derivative and is given by

$$
\begin{gathered}
\text { GCE-I: } \quad\left(1+{ }_{0^{+}}^{R L} D_{t}^{\alpha}\right) J_{R L}(x, t)=-{ }_{0^{+}}^{R L} D_{t}^{1-\beta} \partial_{x} \varrho(x, t), \\
-\partial_{x} J_{R L}(x, t)=\partial_{t} \varrho(x, t),
\end{gathered}
$$

with $\varrho\left(x, 0^{+}\right)=\delta(x), x \in \mathbb{R}, \lim _{|x| \rightarrow \infty} \varrho(x, t)=0$, and $\alpha, \beta \in(0,1]$. The time-fractional Cattaneo equations (2.3) and (2.4) are termed GCE-I with two fractional parameters, where the equivalence between them for the distribution $\varrho(x, t)$ was shown in [2]. Note that we decorate the flux in (1.3) and (2.3) with $C$, and in (1.4) and (2.4) with $R L$ to point out that these equations are not necessarily equivalent for the flux. Note also that both equations (2.3) and (2.4) reduce to (2.1) in the limiting case $\alpha=\beta=1$, and the generalised version of the Cattaneo-type equation of [16] is recovered whenever $\alpha=\beta$. If we only set $\alpha=1$ in equations (2.3) and (2.4), we get

$$
\begin{aligned}
& \text { GCE-III: } \quad\left(1+\partial_{t}\right) J_{R L}(x, t)=-{ }_{0^{+}}^{R L} D_{t}^{1-\beta} \partial_{x} \varrho(x, t) \text {, } \\
& -\partial_{x} J_{R L}(x, t)=\partial_{t} \varrho(x, t),
\end{aligned}
$$

with $\varrho\left(x, 0^{+}\right)=\delta(x), J_{R L}\left(x, 0^{+}\right)=0, x \in \mathbb{R}, \lim _{|x| \rightarrow \infty} \varrho(x, t)=0$, and $\beta \in(0,1]$. Alternatively,

$$
\text { GCE-III: } \begin{array}{r}
\left(1+\partial_{t}\right) J_{C}(x, t)=-\partial_{x} \varrho(x, t), \\
-\partial_{x} J_{C}(x, t)={ }_{0^{+}}^{C} D_{t}^{\beta} \varrho(x, t),
\end{array}
$$

with $\varrho\left(x, 0^{+}\right)=\delta(x), J_{C}\left(x, 0^{+}\right)=0, x \in \mathbb{R}, \lim _{|x| \rightarrow \infty} \varrho(x, t)=0$, and $\beta \in(0,1]$, which are known in the literature as GCE-III. Conversely, if we set $\beta=1$ in (2.3) and (2.4), we obtain the GCE-IV

$$
\text { GCE-IV: }\left(1+{ }_{0^{+}}^{R L} D_{t}^{\alpha}\right) J(x, t)=-\partial_{x} \varrho(x, t), \quad-\partial_{x} J(x, t)=\partial_{t} \varrho(x, t),
$$

with $\varrho\left(x, 0^{+}\right)=\delta(x), x \in \mathbb{R}, \lim _{|x| \rightarrow \infty} \varrho(x, t)=0$, and $\alpha \in(0,1]$, where ${ }_{0^{+}}^{R L} D_{t}^{\alpha}$ can be replaced by ${ }_{0^{+}}^{C} D_{t}^{\alpha}$ and $J\left(x, 0^{+}\right)=0$ should be added to the initial conditions.

Equations (2.5) to (2.7) can be further generalised using the distributed-order derivatives to take the more general forms

$$
\begin{array}{r}
\left(1+\partial_{t}\right) J_{R L}(x, t)=-{ }_{0^{+}}^{R L} D_{t}^{p(1-\nu)} \partial_{x} \varrho(x, t), \\
-\partial_{x} J_{R L}(x, t)=\partial_{t} \varrho(x, t),
\end{array}
$$

with $\varrho\left(x, 0^{+}\right)=\delta(x), J_{R L}\left(x, 0^{+}\right)=0, x \in \mathbb{R}, \lim _{|x| \rightarrow \infty} \varrho(x, t)=0$, and $\sup (p) \subseteq[0,1]$,

$$
\left(1+\partial_{t}\right) J_{C}(x, t)=-\partial_{x} \varrho(x, t), \quad-\partial_{x} J_{C}(x, t)={ }_{0^{+}}^{C} D_{t}^{p(\nu)} \varrho(x, t),
$$


with $\varrho\left(x, 0^{+}\right)=\delta(x), J_{C}\left(x, 0^{+}\right)=0, x \in \mathbb{R}, \lim _{|x| \rightarrow \infty} \varrho(x, t)=0$, and $\sup (p) \subseteq[0,1]$,

$$
\left(1+{ }_{0^{+}}^{R L} D_{t}^{p(\nu)}\right) J(x, t)=-\partial_{x} \varrho(x, t), \quad-\partial_{x} J(x, t)=\partial_{t} \varrho(x, t),
$$

with $\varrho\left(x, 0^{+}\right)=\delta(x), x \in \mathbb{R}, \lim _{|x| \rightarrow \infty} \varrho(x, t)=0$, and $\sup (p) \subseteq[0,1]$, where $a^{+} D_{t}^{p(\nu)}$ and ${ }_{a^{+}}^{R L} D_{t}^{p(\nu)}$ are the distributed-order time-fractional derivatives in the Caputo and Riemann-Liouville senses respectively, defined through

$$
\begin{aligned}
{ }_{a^{+}}^{C} D_{t}^{p(\nu)} f(t) & =\int_{\sup (p)} p(\nu)_{a^{+}}^{C} D_{t}^{\nu} f(t) d \nu, \\
{ }_{a^{+}}^{R L} D_{t}^{p(1-\nu)} f(t) & =\int_{\sup (p)} p(1-\nu)_{a^{+}}^{R L} D_{t}^{\nu} f(t) d \nu \\
& =\int_{\sup (p)} p(\nu)_{a^{+}}^{R L} D_{t}^{1-\nu} f(t) d \nu,
\end{aligned}
$$

and $p(\nu)$ is a probability density function, namely, $p(\nu) \geq 0 \forall \nu \in \sup (p)$, and $\int_{\sup (p)} p(\nu) d \nu=1$. The non-negativity of the distribution $\varrho(x, t)$ of equations (2.3) to (2.10) was discussed in detail in [2. Equation (2.10) was proposed in [1] and analysed in [91] using the multi-term assumption [39].

In this work, we are interested in the case

$$
p(\nu)=p_{1} \delta(\nu-\alpha)+p_{2} \delta(\nu-\beta),
$$

where $0<\alpha<\beta \leq 1$ and $p_{1}+p_{2}=1$. It is salient that when $\alpha=\beta$, or $p_{1}=1, p_{2}=0$, the distributed-order versions of the time-fractional Cattaneo-type equations (2.8) to (2.10) respectively reduce to the generalised Cattaneo equations of type III and IV, equation (2.5) to (2.7). Let us call equations (2.8) to (2.10) with (2.11) and (2.12) the double-order timefractional Cattaneo equations of types I, II, and III. The generic form of the analytical solution for the above Cattaneo-type equations in LaplaceFourier space, (A.5) and (A.6), can be written as

$$
\widehat{\widetilde{\varrho}}(k, s)=\frac{\eta(s) / s}{\eta(s)+k^{2}},
$$

where $\eta(s)$ is the characteristic function to be defined below, and in Laplace space as

$$
\widetilde{\varrho}(x, s)=\frac{\sqrt{\eta(s)}}{2 s} \exp (-|x| \sqrt{\eta(s)})
$$

with $\delta$ th-order moment

$$
\begin{aligned}
\left\langle|x|^{\delta}(s)\right\rangle & =2 \int_{0}^{\infty} x^{\delta} \widetilde{\varrho}(x, s) d x=\frac{\sqrt{\eta(s)}}{s} \int_{0}^{\infty} x^{\delta} \exp (-x \sqrt{\eta(s)}) d x \\
& =\frac{\Gamma(\delta+1)}{s[\eta(s)]^{\delta / 2}}
\end{aligned}
$$


and thus

$$
\left\langle|x|^{\delta}(t)\right\rangle=\Gamma(\delta+1) \mathcal{L}^{-1}\left\{\frac{1}{s[\eta(s)]^{\frac{\delta}{2}}}\right\} .
$$

\section{Analytical solution and mean squared displacement}

Here we provide the exact solutions of the Cattaneo-type equations featured in the previous section, in addition to their corresponding MSDs.

3.1. Generalised Cattaneo equation of type I. In the case of GCEI with two fractional parameters (TFCE), equations (2.3) and (2.4), the characteristic function $\eta(s)$ defined in (2.13) is given by

Thus, we have

$$
\eta(s)=s^{\beta}\left(s^{\alpha}+1\right) \text {. }
$$

$$
\widehat{\widetilde{\varrho}}_{G C E-I}(k, s)=\frac{s^{\beta-1}\left(s^{\alpha}+1\right)}{s^{\beta}\left(s^{\alpha}+1\right)+k^{2}} .
$$

Referring to Problem B.1 in appendix B the solution of equation (3.2) can be extracted from (B.1) by setting $\chi_{0,1}=1$, and $\gamma=\alpha+\beta$, so that we get

$$
\begin{array}{r}
\varrho_{G C E-I}(x, t)=\sum_{n=0}^{\infty} \frac{(-1)^{n}}{n !} t^{\alpha n}\left[\varrho_{1}(x, t)+t^{\alpha} \varrho_{2}(x, t)\right], \\
\varrho_{1}(x, t)=\frac{1}{\sqrt{4 \pi t^{\alpha+\beta}}} H_{1,2}^{2,0}\left[\begin{array}{c|c}
x^{2} & \left(\begin{array}{c}
\left.1+\alpha n-\frac{\alpha+\beta}{2}, \alpha+\beta\right) \\
4 t^{\alpha+\beta}
\end{array}\right. \\
(0,1),\left(\frac{1}{2}+n, 1\right)
\end{array}\right], \\
\varrho_{2}(x, t)=\frac{1}{\sqrt{4 \pi t^{\alpha+\beta}}} H_{1,2}^{2,0}\left[\begin{array}{c|c}
x^{2} & \left(\begin{array}{c}
\left.1+\alpha n+\frac{\alpha-\beta}{2}, \alpha+\beta\right) \\
4 t^{\alpha+\beta}
\end{array}\right] . \\
(0,1),\left(\frac{1}{2}+n, 1\right)
\end{array}\right]
\end{array}
$$

Three subcases of (3.3) can be deduced: the GCE-III $(\alpha=1)$, the GCEIV $(\beta=1)$, and GCE-I with single fractional parameter $(\alpha=\beta)$. We refer to the solution of the space-time fractional Cattaneo equation obtained in [69. which can be considered a special case of (3.3) if the space-fractality is disregarded. Further properties on the space-time Cattaneo equation can be found in [8] and [85].

The $\delta$ th-order moments can be derived by substituting (3.1) into (2.16) and inverting the Laplace transform using (A.19),

$$
\left\langle|x|^{\delta}(t)\right\rangle_{G C E-I}=\Gamma(\delta+1) t^{\frac{\alpha+\beta}{2} \delta} E_{\alpha, \frac{\alpha+\beta}{2} \delta+1}^{\frac{\delta}{2}}\left(-t^{\alpha}\right),
$$

where $E_{\alpha, \beta}^{\gamma}(z)$ is the Prabhakar generalisation of the Mittag-Leffler function (A.16). An equivalent form to (3.4) can be deduced via the Mellin transform of (3.3), see (A.9), and we get 


$$
\begin{aligned}
\left\langle|x|^{\delta}(t)\right\rangle_{G C E-I}= & 2 \int_{0}^{\infty} x^{\delta} \varrho_{G C E-I}(x, t) d x \\
= & 2 \mathcal{M}\left\{\varrho_{G C E-I}(x, t) ; x\right\}(\delta+1) \\
= & 2 \sum_{n=0}^{\infty} \frac{(-1)^{n}}{n !} t^{\alpha n}\left[\mathcal{M}\left\{\varrho_{1}(x, t) ; x\right\}(\delta+1)\right. \\
& \left.\quad+t^{\alpha} \mathcal{M}\left\{\varrho_{2}(x, t) ; x\right\}(\delta+1)\right] \\
= & \frac{2^{\delta}}{\sqrt{\pi}} \Gamma\left(1+\frac{\delta}{2}\right) \Gamma\left(\frac{1+\delta}{2}\right) t^{\frac{\alpha+\beta}{2}} \delta \sum_{n=0}^{\infty}\left\{\frac{\left(1+\frac{\delta}{2}\right)_{n}}{\Gamma\left[\alpha n+\frac{\alpha+\beta}{2} \delta+1\right]} \frac{\left(-t^{\alpha}\right)^{n}}{n !}\right. \\
& \left.\quad+t^{\alpha} \frac{\left(1+\frac{\delta}{2}\right)_{n}}{\Gamma\left[\alpha n+\frac{\alpha+\beta}{2} \delta+\alpha+1\right]} \frac{\left(-t^{\alpha}\right)^{n}}{n !}\right\} \\
= & \frac{2^{\delta}}{\sqrt{\pi}} \Gamma\left(1+\frac{\delta}{2}\right) \Gamma\left(\frac{1+\delta}{2}\right) t^{\frac{\alpha+\beta}{2} \delta} \\
& \times\left[E_{\alpha, \frac{\alpha+\beta}{2} \delta+1}^{\frac{\delta}{2}+1}\left(-t^{\alpha}\right)+t^{\alpha} E_{\alpha, \frac{\alpha+\beta}{2} \delta+\alpha+1}^{\frac{\delta}{2}+1}\left(-t^{\alpha}\right)\right]
\end{aligned}
$$

Both forms of the MSD, (3.4) and (3.5), should be equivalent. This equivalence of (3.4) and (3.5) can be verified by the aid of Legendre's duplication rule and the recurrence relation (A.21). Both forms also have the same limiting behaviour, see (A.17),

$$
\left\langle|x|^{\delta}(t)\right\rangle_{G C E-I} \sim \begin{cases}\frac{2^{\delta}}{\sqrt{\pi}} \frac{\Gamma\left(1+\frac{\delta}{2}\right) \Gamma\left(\frac{1+\delta}{2}\right)}{\Gamma\left(1+\frac{\alpha+\beta}{2} \delta\right)} t^{\frac{\alpha+\beta}{2} \delta}, & t \rightarrow 0^{+} \\ \frac{2^{\delta}}{\sqrt{\pi}} \frac{\Gamma\left(1+\frac{\delta}{2}\right) \Gamma\left(\frac{1+\delta}{2}\right)}{\Gamma\left(1+\frac{\beta}{2} \delta\right)} t^{\frac{\beta}{2} \delta}, & t \rightarrow \infty\end{cases}
$$

and when $\delta=2$, we find the asymptotic behaviour of the MSD,

$$
\left\langle x^{2}(t)\right\rangle_{G C E-I} \sim \begin{cases}\frac{2 t^{\alpha+\beta}}{\Gamma(\alpha+\beta+1)}, & t \rightarrow 0^{+}, \\ \frac{2 t^{\beta}}{\Gamma(\beta+1)}, & t \rightarrow \infty .\end{cases}
$$

The MSD (3.7) generalises the result of GCE-I with one fractional parameter (1.7). It can also represent a retarded subdiffusion process if $0<\alpha+\beta<1$, and a generic crossover from superdiffusion to subdiffusion if $1<\alpha+\beta<2$. The case $0<\alpha+\beta<1$ is analogous to the finding of Chechkin and colleagues [11, 14, 82, 15, 172. On the other hand, the case $1<\alpha+\beta<2$ can depict many phases in [76] for certain pinning forces at low disk activity. The probability density (3.3) and the MSD (3.7) are presented in Figures $1 \mathrm{~T}$ and $1 \mathrm{~b}$ respectively. In Figure 1a, the subdiffusive 
(a)
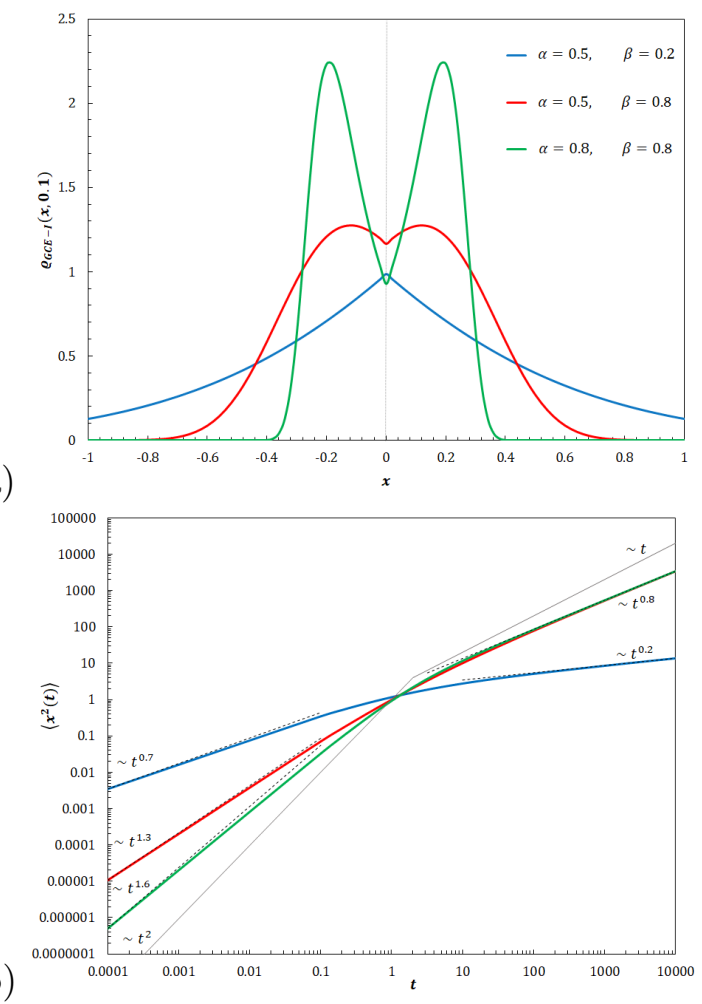

FiguRE 1. (a) Spatial evolution of the probability density $\varrho_{G C E-I}$ of GCE-I at short time $t=0.1$ for different fractional parameters. (b) Temporal evolution of the MSD of GCE-I. The normal and ballistic diffusion limits are shown in the light grey curves.

transition regime in the short-time limiting included in the Cattaneo-type equation is clear in the blue curve, where $0<\alpha+\beta<1$. In the red and green curves, the superdiffusion process is dominant since $1<\alpha+\beta<2$. In Figure 1b the blue curve, i.e., the case corresponding to $\alpha=0.5, \beta=0.2$, the retarded subdiffusion process is salient in the course of time. In the red and green curves of Figure $1 \mathrm{~b}$, which consider the choices $\alpha=0.5, \beta=0.8$ and $\alpha=0.8, \beta=0.8$, namely $1<\alpha+\beta<2$, respectively, the crossover from superdiffusion to subdiffusion in both curves is obvious.

REMARK 3.1. In spite of the generality of the limiting behaviour (3.7) covering a wide range of transitions over the subdiffusive regime or from the superdiffusive to the subdiffusive regime, it is still constrained by the fact that the parameter $\beta$ occurs in both the short-time and the long-time 
behaviours. In essence, the presence of the parameter $\beta$ in the short-time behaviour causes an exemplary transition such as

$$
\left\langle x_{\varrho}^{2}(t)\right\rangle \sim \begin{cases}t^{1.8}, & t \rightarrow 0^{+} \\ t^{0.3}, & t \rightarrow \infty\end{cases}
$$

impossible to be captured by the GCE-I (and thus its special cases; GCE-III and GCE-IV) with two fractional parameters.

REMARK 3.2. It is noteworthy mentioning that GCE-I (3.2) can be written in terms of the generalised diffusion-wave equation with two powerlaw memory kernels [75], namely,

$$
\int_{0}^{t} \xi(t-\tau) \partial_{\tau}^{2} \varrho(x, \tau) d \tau=\partial_{x}^{2} \varrho(x, t),
$$

where $\xi(t)=\frac{t^{1-\beta}}{\Gamma(2-\beta)}+\frac{t^{1-\alpha-\beta}}{\Gamma(2-\alpha-\beta)}, \varrho\left(x, 0^{+}\right)=\delta(x), \partial_{t} \varrho\left(x, 0^{+}\right)=0, \lim _{|x| \rightarrow \infty}$ $\varrho(x, t)=0$, and $\alpha, \beta \in(0,1]$, and in terms of the generalised diffusion-wave equation with regularised Prabhakar derivative, see (A.4),

$$
{\stackrel{\mathcal{P}}{0^{+}}}^{1, \alpha+\beta} D_{\alpha,-1, t} \varrho(x, t)=\partial_{x}^{2} \varrho(x, t),
$$

where $\varrho\left(x, 0^{+}\right)=\delta(x), \partial_{t} \varrho\left(x, 0^{+}\right)=0 \lim _{|x| \rightarrow \infty} \varrho(x, t)=0$, and provided that $0<\alpha, \beta<1$ and $1<\alpha+\beta<2$.

REMARK 3.3. The normalisation of (3.3) can be verified through the Mellin transform,

$$
\begin{aligned}
\int_{-\infty}^{\infty} \varrho_{G C E-I}(x, t) d x=2 \int_{0}^{\infty} \varrho_{G C E-I}(x, t) d x \\
\quad=2 \mathcal{M}\left\{\varrho_{G C E-I}(x, t) ; x\right\}(1) \\
\quad=2 \sum_{n=0}^{\infty} \frac{(-1)^{n}}{n !} t^{\alpha n}\left[\mathcal{M}\left\{\varrho_{1}(x, t) ; x\right\}(1)+t^{\alpha} \mathcal{M}\left\{\varrho_{2}(x, t) ; x\right\}(1)\right] \\
=\sum_{n=0}^{\infty}(-1)^{n}\left[\frac{t^{\alpha n}}{\Gamma(1+\alpha n)}+\frac{t^{\alpha(n+1)}}{\Gamma[1+\alpha(n+1)]}\right]=1
\end{aligned}
$$

3.2. Double-order Cattaneo equation of type I. The double-order time-fractional Cattaneo equation of type I (DTFCE-I) (2.8), (2.11) and (2.12), which is distinguished from the double-order time-fractional diffusion equation of the modified form by the presence of a wave motion in the short-time behaviour, possesses the characteristic function

$$
\eta(s)=\frac{s+1}{p_{1} s^{-\alpha}+p_{2} s^{-\beta}},
$$


where $0<\alpha<\beta \leq 1$ and $p_{1}+p_{2}=1$. For sufficiently large values of time, it can be shown that $\eta(s) \sim\left(p_{1} s^{-\alpha}+p_{2} s^{-\beta}\right)^{-1}$. The solution of DTFCE-I in the Laplace-Fourier space is thus given by (2.13) and (3.10),

$$
\widehat{\widetilde{\varrho}}_{I}(k, s)=\frac{s^{\alpha}+s^{\alpha-1}}{s^{\alpha+1}+s^{\alpha}+k^{2}\left(p_{1}+p_{2} s^{\alpha-\beta}\right)},
$$

which is a special case of Problem B.2, when $\chi_{0,1}=1$, and $\gamma=\alpha+1$. Then, one can deduce that

$$
\varrho_{I}(x, t)=\sum_{n=0}^{\infty} \frac{(-t)^{n}}{n !} \sum_{m=0}^{n}\left(\begin{array}{c}
n \\
m
\end{array}\right)\left[\frac{p_{2}}{p_{1}} t^{\beta-\alpha-1}\right]^{m}\left[\varrho_{1}(x, t)+t \varrho_{2}(x, t)\right],
$$

where

$$
\begin{aligned}
& \varrho_{1}(x, t)=\frac{1}{\sqrt{4 \pi p_{1} t^{\alpha+1}}} \\
& \times H_{2,3}^{2,1}\left[\frac{x^{2}}{4 p_{1} t^{\alpha+1}} \mid \begin{array}{c}
\left(\frac{1}{2}-m, 1\right) ;\left(\frac{1}{2}+n+(\beta-\alpha-1) m-\frac{\alpha}{2}, \alpha+1\right) \\
(0,1),\left(\frac{1}{2}+n-m, 1\right) ;\left(\frac{1}{2}, 1\right)
\end{array}\right], \\
& \varrho_{2}(x, t)=\frac{1}{\sqrt{4 \pi p_{1} t^{\alpha+1}}} \\
& \times H_{2,3}^{2,1}\left[\frac{x^{2}}{4 p_{1} t^{\alpha+1}} \mid \begin{array}{c}
\left(\frac{1}{2}-m, 1\right) ;\left(\frac{3}{2}+n+(\beta-\alpha-1) m-\frac{\alpha}{2}, \alpha+1\right) \\
(0,1),\left(\frac{1}{2}+n-m, 1\right) ;\left(\frac{1}{2}, 1\right)
\end{array}\right]
\end{aligned}
$$

where $p_{1} \geq p_{2}$. It can be verified that the distribution (3.12) is normalised as follows

$$
\begin{aligned}
\int_{-\infty}^{\infty} \varrho_{I}(x, t) d x= & 2 \mathcal{M}\left\{\varrho_{I}(x, t) ; x\right\}(1) \\
= & \sum_{n=0}^{\infty} \frac{(-t)^{n}}{n !} \sum_{m=0}^{n}\left(\begin{array}{c}
n \\
m
\end{array}\right)\left[\frac{p_{2}}{p_{1}} t^{\beta-\alpha-1}\right]^{m} \\
& \times\left\{\frac{\Gamma(1+n-m) \Gamma(m)}{\Gamma(0) \Gamma(1+n+(\beta-\alpha-1) m)}\right. \\
& \left.+\frac{\Gamma(1+n-m) \Gamma(m) \times t}{\Gamma(0) \Gamma(2+n+(\beta-\alpha-1) m)}\right\} .
\end{aligned}
$$

Since $1 / \Gamma(0)=0$, the above series has non-zero value only at $m=0$, then

$$
\int_{-\infty}^{\infty} \varrho_{I}(x, t) d x=\sum_{n=0}^{\infty}(-t)^{n}\left\{\frac{1}{\Gamma(1+n)}+\frac{t}{\Gamma(2+n)}\right\}=1 .
$$

The $\delta$ th- order moments of DTFCE-I can be determined from equations (2.16) and (3.10), 


$$
\begin{aligned}
\left\langle|x|^{\delta}(s)\right\rangle_{I} & =\frac{\Gamma(\delta+1)(s+1)^{-\delta / 2}}{s\left(p_{1} s^{-\alpha}+p_{2} s^{-\beta}\right)^{-\delta / 2}} \\
& =\frac{\Gamma(\delta+1) p_{1}^{\delta / 2} s^{-\frac{\beta+1}{2} \delta-1}\left(1+\frac{1}{s}\right)^{-\delta / 2}}{\left(s^{\beta-\alpha}+\frac{p_{2}}{p_{1}}\right)^{-\delta / 2}} \\
& =\Gamma(\delta+1) p_{1}^{\delta / 2} \sum_{n=0}^{\infty} \frac{(-1)^{n}(\delta / 2)_{n}}{n !} \frac{s^{-\frac{\beta+1}{2} \delta-1-n}}{\left(s^{\beta-\alpha}+\frac{p_{2}}{p_{1}}\right)^{-\delta / 2}},
\end{aligned}
$$

where the expansion $(1+x)^{-r}=\sum_{n=0}^{\infty} \frac{(-1)^{n}(r)_{n}}{n !} x^{n}, \quad r \in \mathbb{R}_{+}, \quad|x|<1$, was employed to $\left(1+\frac{1}{s}\right)^{-\delta / 2}$ for the values $R e(s)>1$. Therefore,

$$
\left\langle|x|^{\delta}(t)\right\rangle_{I}=\Gamma(\delta+1) p_{1}^{\delta / 2} t^{\frac{\alpha+1}{2} \delta} \sum_{n=0}^{\infty} \frac{(-t)^{n}(\delta / 2)_{n}}{n !} E_{\beta-\alpha, \frac{\alpha+1}{2} \delta+n+1}^{-\delta / 2}\left(-\frac{p_{2}}{p_{1}} t^{\beta-\alpha}\right) .
$$

Note that the $\delta$ th-order moments (3.13) represents the exact formula at short and intermediate times time $t$ (since $\operatorname{Re}(s)>1$ ).

The reader can analogously deduce a complementary exact formula for long times by assuming $R e(s)<1$, and expanding then $(1+s)^{-\delta / 2}$ instead of $(1+1 / s)^{-\delta / 2}$. The asymptotic behaviour of all the $\delta$ th-order moments is

$$
\left\langle|x|^{\delta}(t)\right\rangle_{I} \sim \begin{cases}\frac{\Gamma(1+\delta) p_{1}^{\delta / 2}}{\Gamma\left(1+\frac{\alpha+1}{2} \delta\right)} t^{\frac{\alpha+1}{2} \delta}, & t \rightarrow 0^{+} \\ \frac{\Gamma(1+\delta) p_{2}^{\delta / 2}}{\Gamma\left(1+\frac{\beta \delta}{2}\right)} t^{\frac{\beta \delta}{2}}, & t \rightarrow \infty\end{cases}
$$

with MSD limiting behaviours

$$
\left\langle x^{2}(t)\right\rangle_{I} \sim \begin{cases}\frac{2 p_{1} t^{\alpha+1}}{\Gamma(\alpha+2)}, & t \rightarrow 0^{+}, \\ \frac{2 p_{2} t^{\beta}}{\Gamma(\beta+1)}, & t \rightarrow \infty,\end{cases}
$$

for $0<\alpha<\beta \leq 1$, which can be generally viewed as a modified transition from superdiffusion to subdiffusion. It is also included in the wide range of crossovers covered by the generic form (3.7), however, the temporal path on which they evolve is distinct. In the case $0.9<\beta \leq 1$, it represents processes switching from superdiffusion to normal diffusion, whereas the case $0<\alpha \leq 0.1$ describes processes crossing over from normal diffusion to subdiffusion. This type of transition can be found in the transport of electrons in an interacting system subject to quasiperiodic potential [38, specifically, when different interacting strengths $U$ are considered. In Figures $2 \mathrm{a}$ and $2 \mathrm{~b}$ the graphical representations of the distribution (3.12) and 
(a)
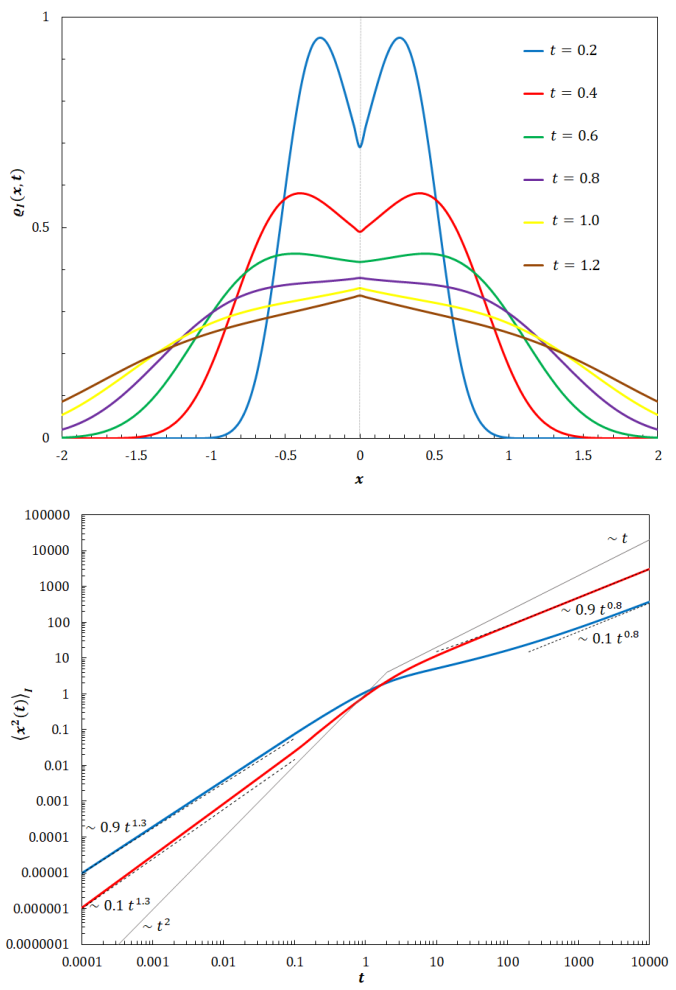

(b)

FiguRE 2. (a) Spatial distribution of DTFCE-I at different instants of time for fractional parameters $\alpha=0.3, \beta=0.8$ and constants $p_{1}=p_{2}=0.5$. (b) MSD of the distribution DTFCE-I versus time for fractional parameters $\alpha=0.3$, $\beta=0.8$ and different values of the model constants; $p_{1}=$ $0.9, p_{2}=0.1$ (blue curve) and $p_{1}=0.1, p_{2}=0.9$ (red curve). The case $p_{1}=p_{2}=0.5$, lying between blue and red curve, is omitted.

MSD of DTFCE-I, $\left\langle x^{2}(t)\right\rangle_{I}$, are shown. In the course of time, the transition from superdiffusion at small times to subdiffusion at longer times is notable in Figure 2a. The apparent warp in the MSD temporal path of Figure 2b seems almost like the result of changing the interacting strength with fixed quasiperiodic potential amplitude, see Figure 2 of [38]. The curvature of this warp can be increased by decreasing the second constant, e.g., $p_{1}=0.99$ and $p_{1}=0.01$.

3.3. Double-order Cattaneo equation of type II. We consider here the double-order time-fractional Cattaneo equation of type II (DTFCE-II), 
(2.9), (2.11) and (2.12). It reduces to the double-order time-fractional diffusion equation of the natural form in the relatively long temporal domain which typifies subdiffusive processes that become more anomalous with time progress. The characteristic function $\eta(s)$ is given as

$$
\eta(s)=(s+1)\left(p_{1} s^{\alpha}+p_{2} s^{\beta}\right),
$$

where $0<\alpha<\beta \leq 1$ and $p_{1}+p_{2}=1$. It appears from (3.16) that $\eta(s) \sim p_{1} s^{\alpha}+p_{2} s^{\beta}$ for relatively large values of time which coincides the characteristic function of the double-order time-fractional diffusion equation of the natural form. In the long time limit, $\eta(s) \sim p_{1} s^{\alpha}+p_{2} s^{\beta} \sim p_{1} s^{\alpha}$ proviso $p_{1} \leq p_{2}$. Upon setting $\eta(s) \sim p_{1} s^{\alpha}$ in (2.13), one can get $\left\langle x^{2}(t)\right\rangle \sim t^{\alpha}$, and vice versa in the short time limit. This result was analytically shown in [11, $82,15,72]$. The distribution of DTFCE-II is a direct consequence of using of (3.16) in (2.13),

$$
\widehat{\widetilde{\varrho}}_{I I}(k, s)=\frac{p_{1} s^{\alpha}+p_{2} s^{\beta}+p_{1} s^{\alpha-1}+p_{2} s^{\beta-1}}{p_{1} s^{\alpha+1}+p_{2} s^{\beta+1}+p_{1} s^{\alpha}+p_{2} s^{\beta}+k^{2}} .
$$

A similar case to (3.17) has been processed in Problem B.3. Thus, setting $\chi_{0,1}=1, \gamma_{1}=\alpha+1$ and $\gamma_{2}=\beta+1$ in (B.3) leads to the solution of DTFCE-II,

$$
\begin{aligned}
& \varrho_{I I}(x, t)=\sum_{n=0}^{\infty} \frac{\left(-t^{\beta-\alpha}\right)^{n}}{n !} \sum_{m=0}^{n}\left(\begin{array}{c}
n \\
m
\end{array}\right)\left(\frac{p_{1}}{p_{2}}\right)^{n-m} t^{(\alpha-\beta-1) m} \\
& \times \sum_{\ell=0}^{m}\left(\begin{array}{c}
m \\
\ell
\end{array}\right)\left[\frac{p_{1}}{p_{2}} t^{\beta-\alpha}\right]^{\ell} \times \\
& \times\left\{\frac{p_{1}}{p_{2}} t^{\beta-\alpha}\left[\varrho_{1}(x, t)+t \varrho_{3}(x, t)\right]+\varrho_{2}(x, t)+t \varrho_{4}(x, t)\right\}, \\
& \varrho_{1}(x, t)=\frac{1}{\sqrt{\frac{4 \pi t^{\beta+1}}{p_{2}}}} \quad(0,1),\left(\frac{1}{2}+n, 1\right) \\
& \times H_{1,2}^{2,0}\left[\frac{p_{2} x^{2}}{4 t^{\beta+1}} \mid\left(\frac{1}{2}+m+(\beta-\alpha)(n-m+\ell+1)-\frac{\beta}{2}, \beta+1\right)\right], \\
& \varrho_{2}(x, t)=\frac{1}{\sqrt{\frac{4 \pi t^{\beta+1}}{p_{2}}}} \quad(0,1),\left(\frac{1}{2}+n, 1\right) \\
& \times H_{1,2}^{2,0}\left[\frac{p_{2} x^{2}}{4 t^{\beta+1}} \mid\left(\frac{1}{2}+m+(\beta-\alpha)(n-m+\ell)-\frac{\beta}{2}, \beta+1\right)\right],
\end{aligned}
$$




$$
\begin{aligned}
& \varrho_{3}(x, t)=\frac{1}{\sqrt{\frac{4 \pi t^{\beta+1}}{p_{2}}}} \\
& \times H_{1,2}^{2,0}\left[\frac{p_{2} x^{2}}{4 t^{\beta+1}} \mid \begin{array}{c}
\left(\frac{3}{2}+m+(\beta-\alpha)(n-m+\ell+1)-\frac{\beta}{2}, \beta+1\right) \\
(0,1),\left(\frac{1}{2}+n, 1\right)
\end{array}\right], \\
& \varrho_{4}(x, t)=\frac{1}{\sqrt{\frac{4 \pi t^{\beta+1}}{p_{2}}}} \\
& \times H_{1,2}^{2,0}\left[\frac{p_{2} x^{2}}{4 t^{\beta+1}} \mid \begin{array}{c}
\left(\frac{3}{2}+m+(\beta-\alpha)(n-m+\ell)-\frac{\beta}{2}, \beta+1\right) \\
(0,1),\left(\frac{1}{2}+n, 1\right)
\end{array}\right],
\end{aligned}
$$

where $p_{1}<p_{2}$. The distribution (3.18) is normalised over $\mathbb{R}$. Indeed,

$$
\begin{aligned}
\int_{-\infty}^{\infty} \varrho_{I I}(x, t) d x= & \sum_{n=0}^{\infty}\left(-t^{\beta-\alpha}\right)^{n} \sum_{m=0}^{n}\left(\begin{array}{c}
n \\
m
\end{array}\right)\left(\frac{p_{1}}{p_{2}}\right)^{n-m} t^{(\alpha-\beta-1) m} \\
& \times \sum_{\ell=0}^{m}\left(\begin{array}{c}
m \\
\ell
\end{array}\right)\left[\frac{p_{1}}{p_{2}} t^{\beta-\alpha}\right]^{\ell} \\
& \times\left\{\frac{\frac{p_{1}}{p_{2}} t^{\beta-\alpha}}{\Gamma(1+m+(\beta-\alpha)(n-m+\ell+1))}\right. \\
& +\frac{\frac{p_{1}}{p_{2}} t^{\beta-\alpha+1}}{\Gamma(2+m+(\beta-\alpha)(n-m+\ell+1))} \\
& +\frac{1}{\Gamma(1+m+(\beta-\alpha)(n-m+\ell))} \\
& \left.+\frac{t}{\Gamma(2+m+(\beta-\alpha)(n-m+\ell))}\right\}=1,
\end{aligned}
$$

which can be attained by expansion for $n=0,1,2,3$. We find that all terms cancel each other except the first term of $1 / \Gamma(1+m+(\beta-\alpha)(n-m+\ell))$.

Following similar procedures to the $\delta$ th-order moments of DTFCE-I, we obtain

$$
\left\langle|x|^{\delta}(t)\right\rangle_{I I}=\frac{\Gamma(\delta+1) t^{\frac{\beta+1}{2}} \delta}{p_{2}^{\delta / 2}} \sum_{n=0}^{\infty} \frac{(-t)^{n}(\delta / 2)_{n}}{n !} E_{\beta-\alpha, \frac{\beta+1}{2} \delta+n+1}^{\delta / 2}\left(-\frac{p_{1}}{p_{2}} t^{\beta-\alpha}\right),
$$

for short- and intermediate- times, with the limiting behaviours

$$
\left\langle|x|^{\delta}(t)\right\rangle_{I I} \sim \begin{cases}\frac{\Gamma(1+\delta)}{p_{2}^{\delta / 2} \Gamma\left(1+\frac{\beta+1}{2} \delta\right)} t^{\frac{\beta+1}{2} \delta}, & t \rightarrow 0^{+} \\ \frac{\Gamma(1+\delta)}{p_{1}^{\delta / 2} \Gamma\left(1+\frac{\alpha \delta}{2}\right)} t^{\frac{\alpha \delta}{2}}, & t \rightarrow \infty\end{cases}
$$


and MSD asymptotes

$$
\left\langle x^{2}(t)\right\rangle_{I I} \sim \begin{cases}\frac{2 t^{\beta+1}}{p_{2} \Gamma(\beta+2)}, & t \rightarrow 0^{+}, \\ \frac{2 t^{\alpha}}{p_{1} \Gamma(\alpha+1)}, & t \rightarrow \infty,\end{cases}
$$

where $0<\alpha<\beta \leq 1$ and $p_{1}+p_{2}=1$. The limiting behaviour (3.21) proves that the DTFCE-II provides a second modified version of the transitions from superdiffusion to subdiffusion. On the other hand, for $0.9<\beta \leq 1$ and $0<\alpha \leq 0.9$, we have a crossover from ballistic behaviour to subdiffusion, which essentially characterises the DTFCE-II. This crossover could not be performed by the generic behaviour (3.7) nor the specific behaviour (3.15). The distribution (3.18) and the MSD of the DTFCE-II are drawn in figures 3a and 3 $\mathrm{b}$, respectively. In accord with the condition $\left(p_{1}<p_{2}\right)$, see (3.18) below, we set $p_{1}=0.4, p_{2}=0.6$ in Figure 3 a. Also, we choose $\alpha=$ $0.3, \beta=0.8$ for closer comparisons with Figure $2 \mathrm{a}$. The DTFCE-II curve remarkably records values for the distribution $\varrho_{I I}(x, t)$ higher than those recorded in figure $2 a$ for DTFCE-I. In the course of time, the transition from superdiffusion at small times to subdiffusion at long times is notable here as well. The presence of $p_{1}$ and $p_{2}$, where $p_{1}+p_{2}=1$, in the MSD covers a significant range in the vicinity of the points $t^{\beta+1}$ and $t^{\alpha}$, see Figure $3 \mathrm{~b}$.

3.4. Double-order Cattaneo equation of type III. In this subsection, we study the effect of distributed-order fractional derivatives on the generalised Cattaneo equation of type IV. The resulting model is termed doubleorder time-fractional Cattaneo equation of type III (DTFCE-III), having the characteristic function

$$
\eta(s)=s\left(1+p_{1} s^{\alpha}+p_{2} s^{\beta}\right),
$$

where $0<\alpha<\beta \leq 1$ and $p_{1}+p_{2}=1$. The long time behaviour of (3.22) is distinctly $\eta(s) \sim s$, which in combination with (2.13) conducts as, the most prominent, the Gaussian distribution, i.e. $\left\langle x^{2}(t)\right\rangle \sim t$. Conversely for short times (3.22) behaves as $\eta(s) \sim s\left(p_{1} s^{\alpha}+p_{2} s^{\beta}\right)$. This form resembles the characteristic function of the double-order timefractional wave equation of natural type [2, 75, 23, which offers decelerating superdiffusive behaviour in the intermediate short-time domain, see Section 3.3 in $[75$. Thus, the study of the characteristic function suggests the progress $\left\langle x^{2}(t)\right\rangle \sim t^{\beta+1} \rightarrow t$ in the course of time.

The characteristic function (3.22) with (2.13) produces the $(k-s)$ form

$$
\widehat{\widetilde{\varrho}}_{I I I}(k, s)=\frac{1+p_{1} s^{\alpha}+p_{2} s^{\beta}}{p_{1} s^{\alpha+1}+p_{2} s^{\beta+1}+s+k^{2}},
$$

which has been considered in a generic form in Problem B.4. Consequently, invoking equation (B.4) after setting $\chi_{0,1}=1, \gamma_{1}=\alpha+1$ and $\gamma_{2}=\beta+1$, helps in getting the exact (conditional) form 
(a)
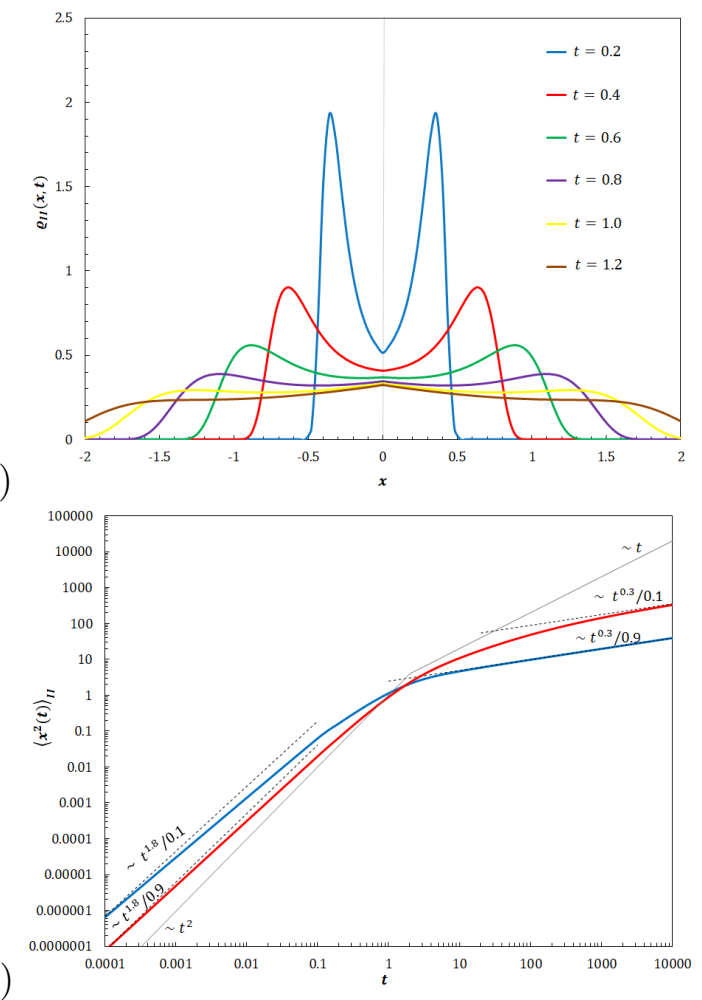

FiguRE 3. (a) Spatial distribution of DTFCE-II at different instants of time for fractional parameters $\alpha=0.3, \beta=$ 0.8 and constants $p_{1}=0.4, p_{2}=0.6$. (b) $\operatorname{MSD}\left\langle x^{2}\right\rangle_{I I}$ of DTFCE-II as a function of time for fractional parameters $\alpha=0.3, \beta=0.8$ and different values of model constants; $p_{1}=0.9, p_{2}=0.1$ (blue curve) and $p_{1}=0.1, p_{2}=0.9$ (red curve).

$$
\begin{aligned}
& \varrho_{I I I}(x, t)=\sum_{n=0}^{\infty} \frac{(-1)^{n}}{n !}\left[\frac{t^{\beta}}{p_{2}}\right]^{n} \sum_{m=0}^{n}\left(\begin{array}{c}
n \\
m
\end{array}\right)\left[\frac{p_{1}}{t^{\alpha}}\right]^{m} \\
& \times\left\{\frac{t^{\beta}}{p_{2}} \varrho_{1}(x, t)+\frac{p_{1}}{p_{2}} t^{\beta-\alpha} \varrho_{2}(x, t)+\varrho_{3}(x, t)\right\}, \\
& \varrho_{1}(x, t)=\frac{1}{\sqrt{4 \pi t^{\beta+1} / p_{2}}} \\
& \times H_{1,2}^{2,0}\left[\frac{x^{2}}{4 t^{\beta+1} / p_{2}} \mid \begin{array}{c}
\left(\frac{1}{2}+\beta n-\alpha m+\frac{\beta}{2}, \beta+1\right) \\
(0,1),\left(\frac{1}{2}+n, 1\right)
\end{array}\right],
\end{aligned}
$$




$$
\begin{aligned}
& \varrho_{2}(x, t)=\frac{1}{\sqrt{4 \pi t^{\beta+1} / p_{2}}}
\end{aligned}
$$

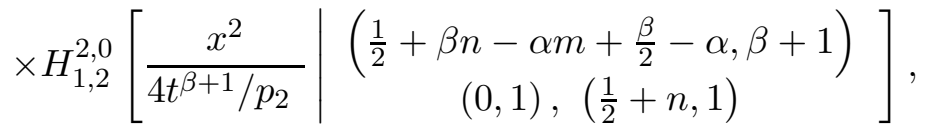

$$
\begin{aligned}
& \varrho_{3}(x, t)=\frac{1}{\sqrt{4 \pi t^{\beta+1} / p_{2}}}
\end{aligned}
$$

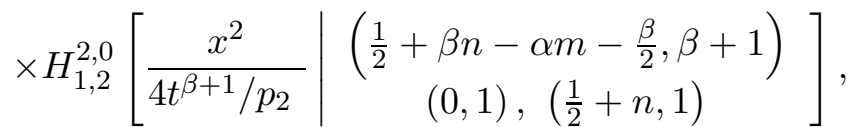

where $p_{1} \in[0,0.3], p_{2} \in[0.7,1], p_{1}+p_{2}=1, \beta \in[0.4,1], 0<\alpha<\beta$. The normalisation of (3.24) can be checked through the expansion of the series

$$
\begin{gathered}
\int_{-\infty}^{\infty} \varrho_{I I I}(x, t) d x=\sum_{n=0}^{\infty}(-1)^{n} \sum_{m=0}^{n}\left(\begin{array}{c}
n \\
m
\end{array}\right)\left\{\frac{\frac{p_{1}^{m}}{p_{2}^{n+1}} t^{\beta(n+1)-\alpha m}}{\Gamma(1+\beta(n+1)-\alpha m)}\right. \\
\left.+\frac{\frac{p_{1}^{m+1}}{p_{2}^{n+1}} t^{\beta(n+1)-\alpha(m+1)}}{\Gamma(1+\beta(n+1)-\alpha(m+1))}+\frac{\frac{p_{1}^{m}}{p_{2}^{n}} t^{\beta n-\alpha m}}{\Gamma(1+\beta n-\alpha m)}\right\}=1 .
\end{gathered}
$$

The scaling properties for the DTFCE-III can be obtained through its $\delta$ thorder moments

$$
\begin{aligned}
\left\langle|x|^{\delta}(t)\right\rangle_{I I I}= & \frac{\Gamma(\delta+1) t^{\frac{\beta+1}{2}} \delta}{p_{2}^{\delta / 2}} \sum_{n=0}^{\infty} \frac{\left(-t^{\beta}\right)^{n}}{n !} \frac{(\delta / 2)_{n}}{p_{2}^{n}} \\
& \times E_{\beta-\alpha, \frac{\beta+1}{2} \delta+\beta n+1}^{n+\delta / 2}\left(-\frac{p_{1}}{p_{2}} t^{\beta-\alpha}\right),
\end{aligned}
$$

for short- and intermediate-time values, with short- and long-time behaviours

$$
\left\langle|x|^{\delta}(t)\right\rangle_{I I I} \sim \begin{cases}\frac{\Gamma(1+\delta)}{p_{2}^{\delta / 2} \Gamma\left(1+\frac{\beta+1}{2} \delta\right)} t^{\frac{\beta+1}{2} \delta}, & t \rightarrow 0^{+}, \\ \frac{\Gamma(1+\delta)}{\Gamma\left(1+\frac{\delta}{2}\right)} t^{\frac{\delta}{2}}, & t \rightarrow \infty .\end{cases}
$$

Hence, the MSD of the DTFCE-III reads

$$
\left\langle x^{2}(t)\right\rangle_{I I I} \sim \begin{cases}\frac{2 t^{\beta+1}}{p_{2} \Gamma(\beta+2)}, & t \rightarrow 0^{+}, \\ 2 t, & t \rightarrow \infty\end{cases}
$$

which confirms the predictions of the characteristic functions (3.22). The domination of the largest exponent in the short-time domain is evident. The behaviour (3.26) represents generally a transition from superdiffusion to strictly normal diffusion. What distinguishes this model from the GCE-IV, in essence, is the presence of another smaller fractional parameter $\alpha$, which 
unfortunately disappears from (3.26), and the constant $p_{2}$ in the short-time limit. This parameter, however, seems to be activated in the short-time domain, which may lead to a retardation in this time scale. In Figure 4 a we represent graphically the probability density function $\varrho_{I I I}(x, t)$ of DTFCEIII at different values of time for the fractional parameters $\alpha=0.3, \beta=0.8$ and model constants $p_{1}=0.3, p_{2}=0.7$, depending on the conditions imposed on the closed-form solution (3.24), and its MSD for fractional parameters $\alpha=0.3, \beta=0.8$ and different values of model constants in Figure 4b. Due to the resemblance between the MSD of DTFCE-II and DTFCE-III in the short-time behaviour, they record higher values in this time domain compared with the DTFCE-I where the smallest exponent $\alpha$ dominates.

\section{Diffusive flux and distribution-like formulation}

In this section we provide an alternative formulation for the Green's function of diffusion (1.1), time-fractional diffusion (1.3) and (1.4), and time-fractional Cattaneo-type diffusion (2.3) and (2.4), by introducing a field variable that behaves like the distribution of the diffusing substance through having the same MSD of $\varrho(x, t)$. The exact formulas for the diffusive flux are also derived.

4.1. Normal diffusion. At first, let us start with the normal diffusion equation (1.1) which can be converted using the transform technique to

$$
\widehat{\widetilde{\varrho}}(k, s)=\frac{1}{s+k^{2}}, \widehat{\widetilde{J}}(k, s)=\frac{-\imath k}{s+k^{2}},
$$

where $\varrho(x, t)$ and $J(x, t)$ have the same partial differential (diffusion) equation $\partial_{x}^{2} f(x, t)=\partial_{t} f(x, t)$ with different initial conditions for the concentration $\varrho\left(x, 0^{+}\right)=\delta(x)$ and diffusive flux $J\left(x, 0^{+}\right)=-u_{1}(x)$, and $u_{1}(x)$ is the unit doublet function, see Section A.4 in Appendix A. Rewriting (4.1) in the form

$$
\widetilde{\varrho}(x, s)=\frac{1}{2 \sqrt{s}} \exp (-|x| \sqrt{s}), \quad \tilde{J}(x, s)=\frac{|x|}{2 x} \exp (-|x| \sqrt{s}),
$$

ensures the non-negativity of $\varrho(x, t)$ and $J(x, t)$. It is clear that $\widetilde{\varrho}(x, \lambda)$ is a completely monotone function for $\lambda>0$, see Section A.5 in Appendix A as a product of two completely monotone functions, and $\int_{-\infty}^{\infty} \widetilde{\varrho}(x, s) d x=1 / s$ which prove that $\varrho(x, t)$ is a probability density function (PDF). On the other hand, evidently, the flux $J(x, t)$ changes its sign according to the sign of $x$, where $\operatorname{sign}(x)=|x| / x$. Physically, the three-dimensional flux $\mathbf{J}(\mathbf{x}, t)$, $\mathbf{x}=\left(x_{1}, x_{2}, x_{2}\right)$, is a vector quantity expressing the rate of movement of diffusing particles through a unit area. So, it is not expected that $J(x, t)$ 
(a)

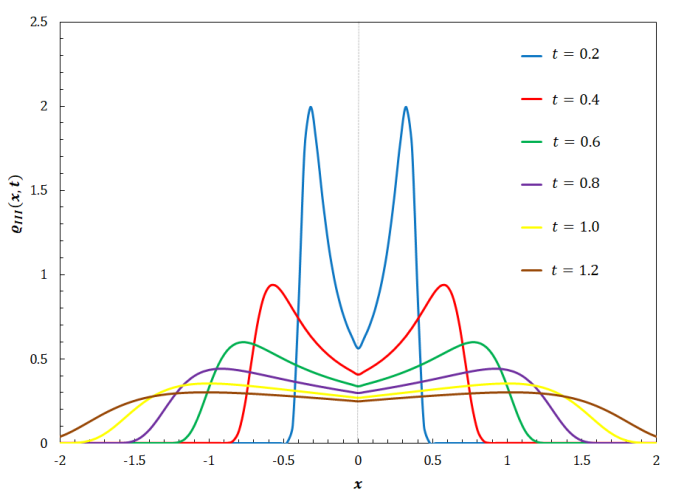

(b)

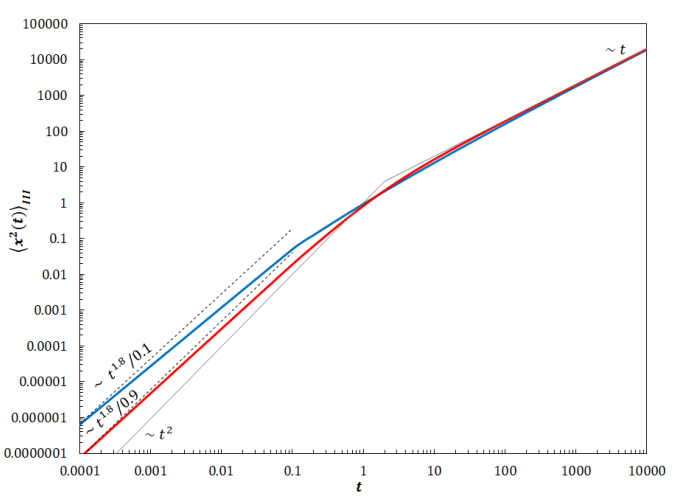

FiguRE 4. (a) Spatial distribution of DTFCE-III at different instants of time for fractional parameters $\alpha=0.3$, $\beta=0.8$ and constants $p_{1}=0.3, p_{2}=0.7$. (b) $\mathrm{MSD}\left\langle x^{2}\right\rangle_{I I I}$ of the DTFCE-III as a function of time for fractional parameters $\alpha=0.3, \beta=0.8$ and different values of model constants; $p_{1}=0.9, p_{2}=0.1$ (blue curve) and $p_{1}=0.1, p_{2}=0.9$ (red curve).

is a PDF. Furthermore, if we retrieve (4.2) in the space-time domain, we get (see also [19])

$$
\varrho(x, t)=\frac{1}{\sqrt{4 \pi t}} \exp \left(-\frac{x^{2}}{4 t}\right), J(x, t)=\frac{x}{2 t \sqrt{4 \pi t}} \exp \left(-\frac{x^{2}}{4 t}\right),
$$

which shows the Gaussian behaviour for particle distribution with $\left\langle x_{\varrho}^{2}(t)\right\rangle \sim$ $t$ and positive skewness for the diffusive flux in the positive half-real axis. The diffusive flux reverses its sign in the negative half-real axis because of the presence of $x$. In other words, the overall flux has to vanish, i.e. $\int_{-\infty}^{\infty} J(x, t) d x=0$. 
Let us consider the quantity

$$
Q(x, t)=x J(x, t)=\frac{x^{2}}{2 t \sqrt{4 \pi t}} \exp \left(-\frac{x^{2}}{4 t}\right)=\frac{x^{2}}{2 t} \varrho(x, t),
$$

which takes the dimensional form $Q^{\prime}\left(x^{\prime}, t^{\prime}\right)=\frac{x^{\prime}}{D} J^{\prime}\left(x^{\prime}, t^{\prime}\right)=-x^{\prime} \partial_{x^{\prime}} \varrho^{\prime}\left(x^{\prime}, t^{\prime}\right)$, where the prime symbols stand for dimensional variables and $D$ is the diffusion coefficient. Hereby, it has the same dimension of $\varrho^{\prime}\left(x^{\prime}, t^{\prime}\right)$, i.e. $\left[Q^{\prime}\right]=\left[\varrho^{\prime}\right]=\mathrm{mol} / \mathrm{cm}^{3}$. Moreover, its Laplace transform is given as

$$
\tilde{Q}(x, s)=\frac{1}{2}|x| \exp (-|x| \sqrt{s}) .
$$

We note that $\tilde{Q}(x, \lambda)$ is a completely monotone function for $\lambda>0$, see definitions (A.1) and (A.4), and $\int_{-\infty}^{\infty} \tilde{Q}(x, s) d x=1 / s$, thereby, $Q(x, t)$ is a PDF. Interestingly, the MSD of $Q(x, t)$ is given by

$$
\left\langle x_{Q}^{2}(t)\right\rangle=6 t=3\left\langle x_{\varrho}^{2}(t)\right\rangle,
$$

namely, $Q$ encodes a behaviour similar to the classical distribution $\varrho(x, t)$ although it possesses a different form, and we call it the "distribution-like".

Using the concept of this distribution-like, we can summarise the main result of this subsection as follows: the classical Fourier-Fick equation (1.1) subject to a Dirac delta initial distribution and unit doublet for the initial flux can be rewritten in the sense of the distribution-like as

$$
Q(x, t)=x J(x, t), \quad x \partial_{x}^{2} J(x, t)=\partial_{t} Q(x, t),
$$

subject to the initial conditions $Q\left(x, 0^{+}\right)=-U_{1}(x)$ and diffusive flux $J\left(x, 0^{+}\right)=-u_{1}(x)$, and boundary condition $\lim _{|x| \rightarrow \infty} Q(x, t)=0$, where $U_{1}(x)$ is the modified unit doublet function, see equations (A.27) and (A.29).

4.2. Time-fractional diffusion. To describe the anomalous diffusive behaviour $\left\langle x_{\varrho}^{2}(t)\right\rangle \sim t^{\beta}, \beta>0$, considerable effort has been invested on the mathematical physics side. Owing to the fact that non-Brownian diffusion is non-universal, today a wide variety of anomalous stochastic processes are known [45. Specifically, fractional diffusion equations were put forward [48, 88, 90, 61], see [40, 43, 59, 28, 27]. Following such ideas, crossovers of the MSD are now frequently described in terms of distributed-order fractional diffusion equations, see the summaries [82, 72, 73]. We here consider single-parameter time-fractional diffusion equations.

Among the single-parameter forms of the time-fractional diffusion equations we pay attention to the two forms (1.3) and (1.4) which result in the same behaviour of the MSD (1.2), $0<\beta \leq 1$, however, they produce two different partial integrodifferential equations for the diffusive flux,

$$
\partial_{x}^{2} J_{R L}(x, t)={ }_{0^{+}}^{R L} D_{t}^{\beta} J_{R L}(x, t)=\partial_{t_{0^{+}}}^{R L} I_{t}^{1-\beta} J_{R L}(x, t),
$$


which requires the initial condition $\lim _{t \rightarrow 0^{+}}{ }_{0^{+}}^{R L} I_{t}^{1-\beta} J_{R L}(x, t)=-u_{1}(x)$, whereas the other reads

$$
\partial_{x}^{2} J_{C}(x, t)={ }_{0^{+}}^{C} D_{t}^{\beta} J_{C}(x, t),
$$

that works well with the conventional initial condition $J\left(x, 0^{+}\right)=-u_{1}(x)$.

REMARK 4.1. It is worth remarking that the RL form (1.4) can be recovered from the form

$$
{ }_{0^{+}}^{R L} I_{t}^{1-\beta} J_{R L}(x, t)=-\partial_{x} \varrho(x, t), \quad-\partial_{x} J_{R L}(x, t)=\partial_{t} \varrho(x, t),
$$

where ${ }_{a^{+}}^{R L} D_{t}^{\beta}$ is the left inverse of $\underset{a^{+}}{R L} I_{t}^{\beta}$, i.e. ${ }_{a^{+}}^{R L} D_{t}^{\beta}\left({ }_{a^{+}}^{R L} I_{t}^{\beta} f(t)\right)=f(t)$, with $\beta>0$. Moreover, the form (4.10) produces the well-known Caputo form $\partial_{x}^{2} \varrho(x, t)={ }_{0^{+}}^{C} D_{t}^{\beta} \varrho(x, t)$ for the distribution and the integrodifferential equation (4.8) for the flux.

Let us now turn our interest to the properties offered by the forms (1.3) and (4.10), where we disregard equation (1.4) because of its equivalence with (59). They possess the transformed distribution

$$
\widehat{\widetilde{\varrho}}(k, s)=\frac{s^{\beta-1}}{s^{\beta}+k^{2}},
$$

which remarkably can be retrieved from (3.2) if we consider the long time behaviour of the characteristic function (3.1), $\eta(s)=s^{\beta}\left(s^{\alpha}+1\right) \sim s^{\beta}$, with equation (2.13), and the transformed fluxes

$$
\widehat{\widetilde{J}}_{C}(k, s)=s^{\beta-1} \widehat{\widetilde{J}}_{R L}(k, s)=-\iota k \frac{s^{\beta-1}}{s^{\beta}+k^{2}} .
$$

By taking the inverse Fourier transform of (4.11) and (4.12) we have

$$
\begin{array}{r}
\widetilde{\varrho}(x, s)=\frac{\sqrt{s^{\beta}}}{2 s} \exp \left(-|x| \sqrt{s^{\beta}}\right), \\
\tilde{J}_{C}(x, s)=s^{\beta-1} \tilde{J}_{R L}(x, s)=\frac{|x|}{2 x} s^{\beta-1} \exp \left(-|x| \sqrt{s^{\beta}}\right) .
\end{array}
$$

It was established that the distribution $\varrho(x, t)$ is a PDF [88] with MSD $\left\langle x_{\varrho}^{2}(t)\right\rangle \sim t^{\beta}$, see also [83] for the subordination technique and [23] for the Bernstein functions technique. It appears also from (4.14) that both fluxes include the sign function, sign $(x)=|x| / x$. We introduce the distributionlike for the time-fractional diffusion equation as

$$
Q(x, t)={ }_{0^{+}}^{R L} I_{t}^{1-\beta}\left[x J_{R L}(x, t)\right]=x J_{C}(x, t),
$$

with its transform

$$
\tilde{Q}(x, s)=\frac{1}{2} s^{\beta-1}|x| \exp \left(-|x| \sqrt{s^{\beta}}\right) .
$$

One can easily check that $\tilde{Q}(x, \lambda) \in \mathcal{C} \mathcal{M F}$ for $\lambda>0$, because it is a product of two completely monotone functions as equation (4.16) 
explains. Also, $\int_{-\infty}^{\infty} \tilde{Q}(x, s) d x=1 / s$ which proves the normalisation of $Q(x, t)$. Thus, the distribution-like of the time-fractional diffusion equations considered in this work is a probability density function. It can be also shown that the MSD of the distribution-like is

$$
\left\langle x_{Q}^{2}(t)\right\rangle=\frac{6 t^{\beta}}{\Gamma(\beta+1)}=3\left\langle x_{\varrho}^{2}(t)\right\rangle,
$$

which likens its counterpart in the normal diffusion case, equation (4.6).

Utilising the definition of distribution-like (4.15), we can rewrite expressions (1.3) and (4.10) in the form

$$
Q(x, t)={ }_{0^{+}}^{R L} I_{t}^{1-\beta}\left[x J_{R L}(x, t)\right], \quad x \partial_{x}^{2} J_{R L}(x, t)=\partial_{t} Q(x, t),
$$

subject to the initial conditions $Q\left(x, 0^{+}\right)=-U_{1}(x)$, the diffusive flux $\lim _{t \rightarrow 0^{+}}{ }_{0^{+}}^{R L} I_{t}^{1-\beta} J_{R L}(x, t)=-u_{1}(x)$, and

$$
Q(x, t)=x J_{C}(x, t), \quad x \partial_{x}^{2} J_{C}(x, t)={ }_{0^{+}}^{C} D_{t}^{\beta} Q(x, t),
$$

$x \partial_{x}^{2} J_{C}(x, t)={ }_{0^{+}}^{C} D_{t}^{\beta} Q(x, t)$ subject to the initial conditions $Q\left(x, 0^{+}\right)=$ $-U_{1}(x)$ and the diffusive flux $J_{C}\left(x, 0^{+}\right)=-u_{1}(x)$ Both equations are subjected to the boundary condition $\lim _{|x| \rightarrow \infty} Q(x, t)=0$. In the case $\beta=1$, equations (4.18) and (4.19) reduce to (4.7). We have shifted the exact formulas for $Q(x, t), J_{R L}(x, t)$ and $J_{C}(x, t)$ to the next subsection to avoid reiteration.

4.3. Time-fractional finite-speed diffusion. The two forms of the timefractional Cattaneo equation describing the finite-speed diffusion were introduced in equations (2.3) and (2.4) in the sense of Caputo and RiemannLiouville fractional derivatives respectively. It was shown that the distribution $\varrho(x, t)$ resulting from (2.3) exactly coincides with the corresponding one based on (2.4), see [2]. Nevertheless, this coincidence is lost when the diffusive flux is considered. In point of fact, in order to mathematically utilise Cattaneo-type equations in studying the itinerant (non-scaling) anomalous behaviour, the initial conditions should be imposed on the rate of the diffusive flux, not the diffusing flux itself. Loosely speaking, equation (2.1) should be subject to $\partial_{t} J\left(x, 0^{+}\right)=-u_{1}(x)$, equation (2.3) subject to $\lim _{t \rightarrow 0^{+}}{ }_{0^{+}}^{C} D_{t}^{\alpha} J_{C}(x, t)=-u_{1}(x)$, and equation (2.4) subject to $\lim _{t \rightarrow 0^{+}}{ }_{0^{+}}^{R L} D_{t}^{\alpha R L} 0_{t}^{1-\beta} J_{R L}(x, t)=-u_{1}(x)$ analogously to its counterpart in the time-fractional diffusion equation. These conditions are all leading to zero initial flux or $\lim _{t \rightarrow 0^{+}}{ }_{0^{+}}^{R L} I_{t}^{1-\beta} J_{R L}(x, t)=0$. In analogue to remark 4.1, we suggest another form for the time fractional Cattaneo equation.

REMARK 4.2. The following Cattaneo-type equation 


$$
\left(1+{ }_{0^{+}}^{R L} D_{t}^{\alpha}\right){ }_{0^{+}}^{R L} I_{t}^{1-\beta} J_{R L}(x, t)=-\partial_{x} \varrho(x, t), \quad-\partial_{x} J_{R L}(x, t)=\partial_{t} \varrho(x, t),
$$

with $\varrho\left(x, 0^{+}\right)=\delta(x), \lim _{t \rightarrow 0^{+}}{ }_{0^{+}}^{R L} I_{t}^{1-\beta} J_{R L}(x, t)=0, x \in \mathbb{R}$, as well as $\lim _{|x| \rightarrow \infty} \varrho(x, t)=0$, and $\alpha, \beta \in(0,1]$, yields the same distribution and diffusive flux provided by (2.4). The condition $\lim _{t \rightarrow 0^{+}}{ }_{0^{+}}^{R L} I_{t}^{1-\beta} J_{R L}(x, t)=0$ guarantees the commutation ${ }_{0^{+}}^{R L} D_{t}^{1-\beta}\left[\begin{array}{l}R L \\ 0^{+}\end{array} D_{t}^{\alpha} f(t)\right]={ }_{0^{+}}^{R L} D_{t}^{\alpha}\left[\begin{array}{l}R L \\ 0^{+}\end{array} D_{t}^{1-\beta} f(t)\right]$. Alternatively, it can be written as $\lim _{t \rightarrow 0^{+}}{ }_{0^{+}}^{R L} D_{t}^{\alpha R L}{0^{+}}_{t}^{1-\beta} J_{R L}(x, t)=-u_{1}(x)$.

Employing the transform technique to equations (2.3) and (4.20) we obtain

in the Laplace-Fourier domain, and

$$
\widehat{\widetilde{J}}_{C}(k, s)=s^{\beta-1} \widehat{\widetilde{J}}_{R L}(k, s)=-\iota k \frac{s^{\beta-1}}{s^{\alpha+\beta}+s^{\beta}+k^{2}},
$$

$$
\tilde{J}_{C}(k, s)=s^{\beta-1} \tilde{J}_{R L}(x, s)=\frac{|x|}{2 x} s^{\beta-1} \exp \left(-|x| \sqrt{s^{\beta}\left(1+s^{\alpha}\right)}\right)
$$

in the Laplace domain. Accordingly, we take the distribution-like of the time-fractional Cattaneo equation in the form

$$
Q(x, t)=\left(1+{ }_{0^{+}}^{R L} D_{t}^{\alpha}\right){ }_{0^{+}}^{R L} I_{t}^{1-\beta}\left[x J_{R L}(x, t)\right]=\left(1+{ }_{0^{+}}^{C} D_{t}^{\alpha}\right)\left[x J_{C}(x, t)\right],
$$

whose Laplace transform is

$$
\tilde{Q}(x, s)=\frac{1}{2} s^{\beta-1}\left(1+s^{\alpha}\right)|x| \exp \left(-|x| \sqrt{s^{\beta}\left(1+s^{\alpha}\right)}\right) .
$$

We now claim that $Q(x, t)$ given by (4.23) is a probability density function if $\alpha+\beta \leq 1$, where $\alpha, \beta \in(0,1]$. To justify this claim, we first prove the normalisation of $Q(x, t)$ by integrating (4.24) over $\mathbb{R}, \int_{-\infty}^{\infty} \tilde{Q}(x, s) d x=1 / s$, namely, $Q(x, t)$ is normalised. Secondly, we study the non-negativity of $Q(x, t)$ by rewriting (4.24) as

$$
\begin{aligned}
& \tilde{Q}(x, \lambda)=\frac{1}{2} f_{1}(\lambda) f_{2}(\lambda) f_{3}(\lambda ;|x|), \lambda>0, \\
& f_{1}(\lambda)=\lambda^{\beta-1}, \\
& f_{2}(\lambda)=\lambda^{\alpha+\beta-1}, \\
& f_{3}(\lambda ;|x|)=|x| \exp \left[-|x|\left(\lambda^{\beta}\right)^{\frac{1}{2}}\left(1+\lambda^{\alpha}\right)^{\frac{1}{2}}\right] .
\end{aligned}
$$

Obviously, $f_{1}(\lambda)$ is completely monotone for $\beta \in(0,1]$ whilst $f_{2}(\lambda)$ is completely monotone for $\alpha+\beta \in(0,1]$, refer to definitions (A.1) and (A.3). On the other hand, we have that $\lambda^{\beta}$ and $1+\lambda^{\alpha}$ are complete Bernstein functions $\alpha, \beta \in(0,1]$ as definition (A.4) reports, note that $1 \in \mathcal{S F} \cap$ 
$\mathcal{C B F}$. Therefore, $\left(\lambda^{\beta}\right)^{\frac{1}{2}}\left(1+\lambda^{\alpha}\right)^{\frac{1}{2}} \in \mathcal{C B F}$, and $f_{3}(\lambda ;|x|) \in \mathcal{C} \mathcal{M F}$. This proves that $\tilde{Q}(x, \lambda)$ is completely monotone as a product of three complete monotone functions. Hence $Q(x, t) \geq 0$ if $\alpha+\beta \leq 1$ and $\alpha, \beta \in(0,1]$, which completes the proof of our claim. On the other hand, this result can be reinforced by verifying that the process following the distribution-like (4.23) is subordinated to the normal (Gaussian) diffusion provided that $\alpha+\beta \leq 1$ and $\alpha, \beta \in(0,1]$, using the following criterion [83, see also [5]. Firstly, equation (4.24) can be rewritten in the form:

$$
\begin{aligned}
\tilde{Q}(x, s) & =-\frac{x}{2 \pi} \partial_{x} \int_{-\infty}^{\infty} \frac{s^{\beta-1}\left(1+s^{\alpha}\right)}{s^{\alpha+\beta}+s^{\beta}+k^{2}} e^{-\iota k x} d k \\
& =-\frac{x}{2 \pi} \partial_{x} \int_{-\infty}^{\infty} s^{\beta-1}\left(1+s^{\alpha}\right)\left\{\int_{0}^{\infty} e^{-\left[s^{\beta}\left(1+s^{\alpha}\right)+k^{2}\right] u} d u\right\} e^{-\imath k x} d k \\
& =-\frac{x}{2 \pi} \partial_{x} \int_{-\infty}^{\infty}\left[\int_{0}^{\infty} e^{-k^{2} u} \tilde{G}(u, s) d u\right] e^{-\iota k x} d k \\
& =\int_{0}^{\infty} \frac{x^{2}}{2 u \sqrt{4 \pi u}} e^{-\frac{x^{2}}{4 u}} \tilde{G}(u, s) d u,
\end{aligned}
$$

where $\tilde{G}(u, s)$, given by

$$
\tilde{G}(u, s)=s^{\beta-1}\left(1+s^{\alpha}\right) e^{-s^{\beta}\left(1+s^{\alpha}\right) u},
$$

is the Laplace transform of the function $G(u, t)$ providing the subordination transformation from the time scale $t$ to the time scale $u$. It remains to prove that $G(u, t)$ is a PDF with respect to $u>0$ for any $t>0$. Firstly, we note that $G(u, t)$ is normalised with respect to $u$. Indeed, since $\int_{0}^{\infty} \tilde{G}(u, s) d u=$ $1 / s$, then $\int_{0}^{\infty} G(u, t) d u=1$. Secondly. The non-negativity of $G(u, t)$ can be proved by verifying that its Laplace transform (4.27) is completely monotone. By rewriting $\tilde{G}(u, s)$ as

$$
\begin{aligned}
& \tilde{G}(u, \lambda)=f_{1}(\lambda) f_{2}(\lambda) g_{1}(u, \lambda) g_{2}(u, \lambda), \lambda>0 \\
& f_{1}(\lambda)=\lambda^{\beta-1} \\
& f_{2}(\lambda)=\lambda^{\alpha+\beta-1} \\
& g_{1}(u, \lambda)=e^{-u \lambda^{\beta}} \\
& g_{2}(u, \lambda)=e^{-u \lambda^{\alpha+\beta}},
\end{aligned}
$$

one concludes that $f_{1}(\lambda)$ and $g_{1}(u, \lambda)$ are completely monotone functions for $\lambda>0$ and $\beta \in(0,1]$, whilst $f_{2}(\lambda)$ and $g_{2}(u, \lambda)$ require that $\lambda>0$, $\alpha, \beta \in(0,1]$ and $\alpha+\beta \leq 1$ to be complete monotone functions, where subsection A.5 of Appendix $\mathrm{A}$ has been invoked repeatedly. Therefore, $\tilde{G}(u, \lambda)$, as a product of complete monotone functions, is in turn a completely monotone, which proves the non-negativity of $G(u, t)$. In view of 
equation (4.4) and the Laplace inversion of (4.26), we can state that the time-fractional Cattaneo equation with two fractional derivatives of different orders $\alpha, \beta \in(0,1]$ represents a mathematical model for a stochastic process subordinated to Gaussian diffusion provided that the fractional derivative orders satisfy $\alpha+\beta \leq 1$. It is worth mentioning that the same criterion can be implemented for the distribution $\varrho(x, t)$ using the same transformation $G(u, t)$.

The mathematical definition of the distribution-like (4.23) enables us to replace the time-fractional Cattaneo equation in the RL-sense (2.4) and (4.20) by

$Q(x, t)=\left(1+\chi_{0,1} 0_{0^{+}}^{R L} D_{t}^{\alpha}\right){ }_{0^{+}}^{R L} I_{t}^{1-\beta}\left[x J_{R L}(x, t)\right], \quad x \partial_{x}^{2} J_{R L}(x, t)=\partial_{t} Q(x, t)$

subject to the initial conditions $Q\left(x, 0^{+}\right)=-U_{1}(x)$ and diffusive flux $\lim _{t \rightarrow 0^{+}}{ }_{0^{+}}^{R L} D_{t}^{\alpha R L} 0^{+} I_{t}^{1-\beta} J_{R L}(x, t)=-u_{1}(x)$, and the time-fractional Cattaneo equation in the C-sense (2.3) by

$$
Q(x, t)=\left(1+\underset{0,1_{0^{+}}^{C}}{C} D_{t}^{\alpha}\right)\left[x J_{C}(x, t)\right], \quad x \partial_{x}^{2} J_{C}(x, t)={ }_{0^{+}}^{C} D_{t}^{\beta} Q(x, t)
$$

subject to the initial conditions $Q\left(x, 0^{+}\right)=-U_{1}(x)$ and diffusive flux $\lim _{t \rightarrow 0^{+}}{ }_{0^{+}}^{C} D_{t}^{\alpha} J_{C}(x, t)=-u_{1}(x), \chi_{0,1} \in\{0,1\}$, and both equations are subject to the boundary condition $\lim _{|x| \rightarrow \infty} Q(x, t)=0$. When $\chi_{0,1}=0$ and $\lim _{\alpha \rightarrow 0^{+}}{ }_{0^{+}}^{C} D_{t}^{\alpha}=\lim _{\alpha \rightarrow 0^{+}}{ }_{0^{+}}^{R L} D_{t}^{\alpha}=1$, equations (4.29) and (4.30) reduce to (4.18) and (4.19), respectively.

Lastly, we complete this section by providing analytical solutions for the diffusive fluxes $J_{R L}(x, t)$ and $J_{C}(x, t)$, and the distribution-like $Q(x, t)$ for TFCE. The diffusive fluxes in RL and $\mathrm{C}$ sense are given by

$$
J_{R L}(x, t)=-\partial_{x} \varphi_{R L}(x, t), \quad J_{C}(x, t)=-\partial_{x} \varphi_{C}(x, t),
$$

where $\varphi_{R L}(x, t)$ and $\varphi_{C}(x, t)$ are given by

$$
\widehat{\widetilde{\varphi}}_{R L}(k, s)=\frac{1}{s^{\gamma}+\chi_{0,1} s^{\beta}+k^{2}}, \quad \widehat{\widetilde{\varphi}}_{C}(k, s)=\frac{s^{\gamma-1}}{s^{\gamma}+\chi_{0,1} s^{\beta}+k^{2}},
$$

where $\chi_{0,1} \in\{0,1\}$, and $\gamma \geq \beta$ such that $\gamma=\beta$ if $\chi_{0,1}=0$ and $\gamma>\beta$ if $\chi_{0,1}=1$. By following the same procedures of solving Problem B.1, one derives

$$
\varphi_{R L}(x, t)=\frac{t^{\gamma-1}}{\sqrt{4 \pi t^{\gamma}}} \sum_{n=0}^{\infty} \frac{\left(-\chi_{0,1}\right)^{n}}{n !} t^{(\gamma-\beta) n} H_{1,2}^{2,0}\left[\frac{x^{2}}{4 t^{\gamma}} \mid \begin{array}{c}
\left((\gamma-\beta) n+\frac{\gamma}{2}, \gamma\right) \\
(0,1),\left(\frac{1}{2}+n, 1\right)
\end{array}\right] .
$$

Then, substituting (4.33) into (4.31) and using (A.13), we arrive at 


$$
J_{R L}(x, t)=\frac{t^{\gamma-1}}{x \sqrt{\pi t^{\gamma}}} \sum_{n=0}^{\infty} \frac{\left(-\chi_{0,1}\right)^{n}}{n !} t^{(\gamma-\beta) n} H_{1,2}^{2,0}\left[\frac{x^{2}}{4 t^{\gamma}} \mid \begin{array}{c}
\left((\gamma-\beta) n+\frac{\gamma}{2}, \gamma\right) \\
(1,1),\left(\frac{1}{2}+n, 1\right)
\end{array}\right]
$$

where the relation $\Gamma(z / 2) \Gamma(1-z) / \Gamma(-z)=-2 \Gamma(1+z / 2)$ has been tacitly utilised. We have two special cases included in equation (4.34): First, when $\chi_{0,1}=0$, equation (4.34) presents the flux of time-fractional diffusion equation (1.4) and (4.10),

$$
J_{R L}^{T F D E}(x, t)=\frac{t^{\beta-1}}{x \sqrt{\pi t^{\beta}}} H_{1,2}^{2,0}\left[\frac{x^{2}}{4 t^{\beta}} \begin{array}{c}
\left(\frac{\beta}{2}, \beta\right) \\
(1,1),\left(\frac{1}{2}, 1\right)
\end{array}\right],
$$

and when $\beta=1$, we get the physical flux of the normal diffusion (1.1),

$$
\begin{aligned}
J^{N D}(x, t) & =\frac{1}{x \sqrt{\pi t}} H_{1,2}^{2,0}\left[\frac{x^{2}}{4 t} \mid \begin{array}{c}
\left(\frac{1}{2}, 1\right) \\
(1,1),\left(\frac{1}{2}, 1\right)
\end{array}\right] \\
& =\frac{1}{x \sqrt{\pi t}} H_{0,1}^{1,0}\left[\frac{x^{2}}{4 t} \mid(1,1)\right] \\
& =\frac{x}{2 t \sqrt{4 \pi t}} \exp \left(-\frac{x^{2}}{4 t}\right),
\end{aligned}
$$

which coincides with equation (4.3). Here, we have used the Mellin transform with equation (A.15). Secondly, when $\chi_{0,1}=1$, then equation (4.34) yields the flux of time-fractional Cattaneo equation (2.4) and (4.20),

$$
\begin{aligned}
J_{R L}^{T F C E}(x, t)= & \frac{t^{\alpha+\beta-1}}{x \sqrt{\pi t^{\alpha+\beta}}} \sum_{n=0}^{\infty} \frac{(-1)^{n}}{n !} t^{\alpha n} \\
& \times H_{1,2}^{2,0}\left[\frac{x^{2}}{4 t^{\alpha+\beta}} \mid \begin{array}{c}
\left(\alpha n+\frac{\alpha+\beta}{2}, \alpha+\beta\right) \\
(1,1),\left(\frac{1}{2}+n, 1\right)
\end{array}\right] .
\end{aligned}
$$

Analogously, we can deduce an exact generic formula for $J_{C}(x, t)$,

$$
\begin{aligned}
J_{C}(x, t)= & \frac{1}{x \sqrt{\pi t \gamma}} \sum_{n=0}^{\infty} \frac{\left(-\chi_{0,1}\right)^{n}}{n !} t^{(\gamma-\beta)(n+1)} \\
& \times H_{1,2}^{2,0}\left[\begin{array}{c|c}
\frac{x^{2}}{4 t^{\gamma}} & \left(1+(\gamma-\beta)(n+1)-\frac{\gamma}{2}, \gamma\right) \\
(1,1),\left(\frac{1}{2}+n, 1\right)
\end{array}\right],
\end{aligned}
$$

which reduces to the flux of TFDE with time-fractality in the continuity equation (1.3) when $\chi_{0,1}=0$,

$$
J_{C}^{T F D E}(x, t)=\frac{1}{x \sqrt{\pi t^{\beta}}} H_{1,2}^{2,0}\left[\frac{x^{2}}{4 t^{\beta}} \mid \begin{array}{c}
\left(1-\frac{\beta}{2}, \beta\right) \\
(1,1),\left(\frac{1}{2}, 1\right)
\end{array}\right] .
$$


(a)

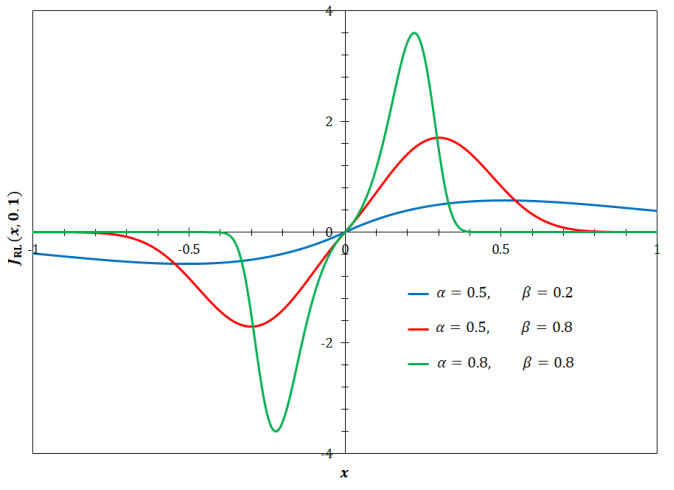

(b)

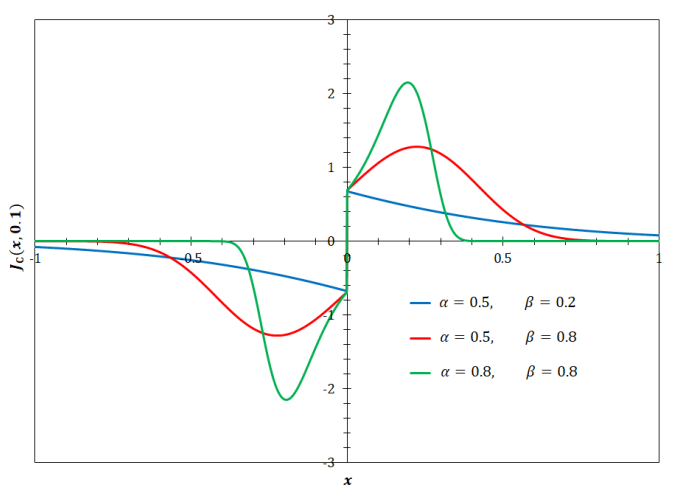

FiguRE 5. (a) Diffusive flux $J_{R L}^{T F C E}$ (without fractality in the continuity equation) at dimensionless time $t=0.1$, and different fractional parameters. (b) Diffusive flux $J_{C}^{T F C E}$ (with fractality in the continuity equation) at dimensionless time $t=0.1$, and different fractional parameters.

Note that $J_{C}^{T F D E}(x, t)=J_{R L}^{T F D E}(x, t)=J^{N D}(x, t)$ in the case $\beta=1$. Conversely, when $\chi_{0,1}=1$, equation (4.37) reduces to the flux of TFCE with time-fractality in the continuity equation (2.3),

$$
\begin{aligned}
J_{C}^{T F C E}(x, t)= & \frac{t^{\alpha}}{x \sqrt{\pi t^{\alpha+\beta}}} \sum_{n=0}^{\infty} \frac{\left(-t^{\alpha}\right)^{n}}{n !} \\
& \times H_{1,2}^{2,0}\left[\frac{x^{2}}{4 t^{\alpha+\beta}} \mid \begin{array}{c}
\left(1+\alpha n+\frac{\alpha-\beta}{2}, \alpha+\beta\right) \\
(1,1),\left(\frac{1}{2}+n, 1\right)
\end{array}\right]
\end{aligned}
$$

The diffusive fluxes in the RL sense (without fractality in the continuity equation) (4.36), and in the $\mathrm{C}$ sense (with temporal fractality in the continuity equation) (4.39) are drawn in figures 5a and 50, respectively. 
Finally, the distribution-like can be directly derived from the unified nondimensional relation

$$
Q(x, t)=-x \partial_{x} \varrho(x, t)
$$

together with the universal distribution (B.1), we get, upon using (A.12) and (A.13), the Mellin transform and the known relation of the Gamma function, $\Gamma(z / 2) \Gamma(1-z) / \Gamma(-z)=-2 \Gamma(1+z / 2)$,

$$
\begin{aligned}
& Q(x, t)=\sum_{n=0}^{\infty} \frac{\left(-\chi_{0,1}\right)^{n}}{n !} t^{(\gamma-\beta) n}\left[Q_{1}(x, t)+\chi_{0,1} t^{\gamma-\beta} Q_{2}(x, t)\right], \\
& Q_{1}(x, t)=\frac{1}{\sqrt{\pi t^{\gamma}}} H_{1,2}^{2,0}\left[\frac{x^{2}}{4 t^{\gamma}} \mid \begin{array}{c}
\left(1+(\gamma-\beta) n-\frac{\gamma}{2}, \gamma\right) \\
(1,1),\left(\frac{1}{2}+n, 1\right)
\end{array}\right] \text {, } \\
& Q_{2}(x, t)=\frac{1}{\sqrt{\pi t^{\gamma}}} H_{1,2}^{2,0}\left[\frac{x^{2}}{4 t^{\gamma}} \mid \begin{array}{c}
\left(1+(\gamma-\beta)(n+1)-\frac{\gamma}{2}, \gamma\right) \\
(1,1),\left(\frac{1}{2}+n, 1\right)
\end{array}\right] .
\end{aligned}
$$

Again, the last generic formula for the distribution-like contains two special cases of interest: the first case is $\chi_{0,1}=0$, that gives the distribution-like for TFDE (4.18) and (4.19)

$$
Q^{T F D E}(x, t)=\frac{1}{\sqrt{\pi t^{\beta}}} H_{1,2}^{2,0}\left[\begin{array}{l|c}
x^{2} & \left(1-\frac{\beta}{2}, \beta\right) \\
4 t^{\beta} & (1,1),\left(\frac{1}{2}, 1\right)
\end{array}\right],
$$

which reduces to (4.4) in the limit $\beta=1$. Meanwhile, the second case, $\chi_{0,1}=1$, gives the distribution-like for TFCE (4.29) and (4.30)

$$
\begin{aligned}
& Q^{T F C E}(x, t)=\sum_{n=0}^{\infty} \frac{(-1)^{n}}{n !} t^{\alpha n}\left[Q_{1}(x, t)+t^{\alpha} Q_{2}(x, t)\right], \\
& Q_{1}(x, t)=\frac{1}{\sqrt{\pi t^{\alpha+\beta}}} H_{1,2}^{2,0}\left[\begin{array}{c|c}
x^{2} & \left(\begin{array}{c}
\left.1+\alpha n-\frac{\alpha+\beta}{2}, \alpha+\beta\right) \\
4 t^{\alpha+\beta}
\end{array}\right. \\
(1,1),\left(\frac{1}{2}+n, 1\right)
\end{array}\right], \\
& Q_{2}(x, t)=\frac{1}{\sqrt{\pi t^{\alpha+\beta}}} H_{1,2}^{2,0}\left[\begin{array}{c|c}
\frac{x^{2}}{4 t^{\alpha+\beta}} & \left(\begin{array}{c}
\left.1+\alpha n+\frac{\alpha-\beta}{2}, \alpha+\beta\right) \\
(1,1),\left(\frac{1}{2}+n, 1\right)
\end{array}\right]
\end{array}\right.
\end{aligned}
$$

for $\alpha+\beta \leq 1$, see Figures 6 and $6 \mathrm{~b}$ for the graphical representations of the closed-form (4.43). The subordination of the process governed by TFCE to the Gaussian process is verified whenever the distribution-like $Q(x, t)$ is non-negative. In the green curve of Figure $6 a$ the non-negativity condition has been clearly breached due to the choice $1<\alpha+\beta<2$ violating the condition $\alpha+\beta<1$ established above. It comes to our attention that the general form of distribution-like (4.41) can be derived by using the Laplace transform of equations (4.23), (4.34) and (4.37) and utilising the useful relations (2.20) and (2.21) in 60]. As applied in previous sections, the Mellin transform can be employed here to check the normalisation of (4.43) 
(a)
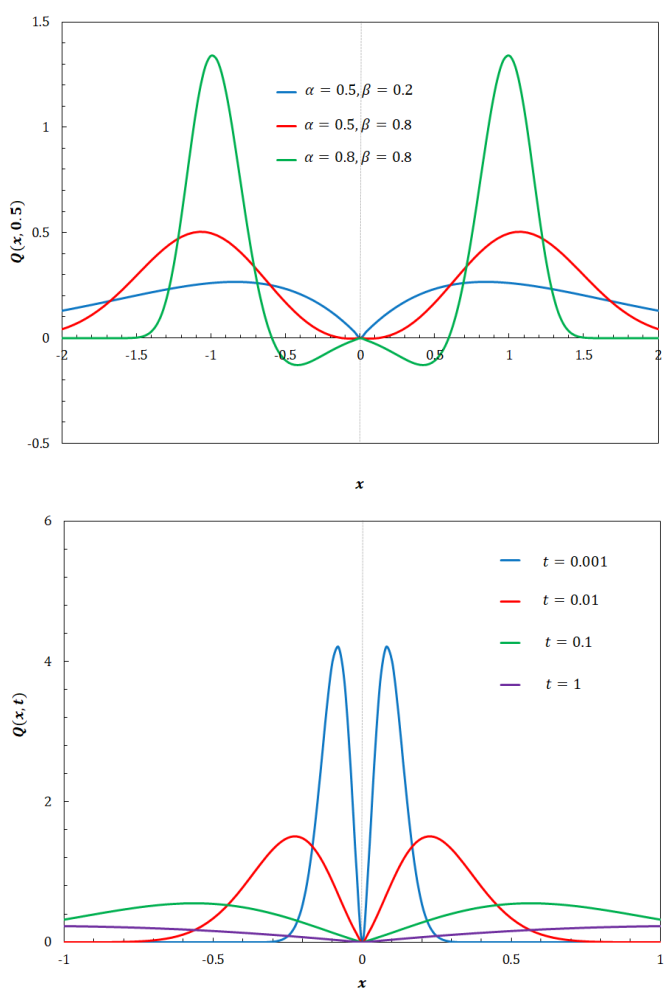

(b)

FiguRE 6. (a) Spatial evolution of the distribution-like $Q(x, t)$ of the time-fractional Cattaneo-type equation with two fractional parameters at dimensionless time $t=0.5$. (b) Spatial distribution of $Q(x, t)$ of TFCE subordinating to the Gaussian process (non-negative) at different instants of time and fractional parameters $\alpha=0.5, \beta=0.4$.

and obtain the $\delta$ th-order moment of $Q^{T F C E}(x, t)$, one can obtain the most interesting result,

$$
\left\langle\left|x_{Q}\right|^{\delta}(t)\right\rangle=(\delta+1)\left\langle\left|x_{\varrho}\right|^{\delta}(t)\right\rangle,
$$

which suggests an alternative definition for the distribution of the diffusing substance with alternative governing equations (4.29) and (4.30) for time-fractional finite-speed diffusion, (4.18) and (4.19) for time-fractional diffusion, and (4.7) for the Gaussian diffusion.

REMARK 4.3. The relation between the fluxes, (4.36) and (4.39) and the distribution-like (4.43) can be rewritten in the integral forms 


\begin{tabular}{|c|c|c|}
\hline Model [resp. equations] & $\left\langle x^{2}(t)\right\rangle$ & $\left\langle x^{2}(t)\right\rangle$ \\
& $t \rightarrow 0+$ & $t \rightarrow \infty$ \\
\hline 2-parameter TFCE/GCE-I [(2.3) $,(2.4),([4.20)]$ & $\sim t^{\alpha+\beta}$ & $\sim t^{\beta}$ \\
\hline DTFCE-I [(2.8), (2.11), (2.12)] & $\sim p_{1} t^{\alpha+1}$ & $\sim p_{2} t^{\beta}$ \\
\hline DTFCE-II [(2.9) $,(2.11),(2.12)]$ & $\sim t^{\beta+1} / p_{2}$ & $\sim t^{\alpha} / p_{1}$ \\
\hline DTFCE-III [(2.10) $,(2.11),(2.12)]$ & $\sim t^{\beta+1} / p_{2}$ & $\sim t$ \\
\hline
\end{tabular}

TABLE 1. Limiting behaviour of the MSD for the proposed Cattaneo-like models, along with the respective equation numbers.

$$
\begin{aligned}
& J_{R L}^{T F C E}(x, t)=\frac{1}{x} \int_{0}^{t} \frac{Q^{T F C E}(x, \tau)}{(t-\tau)^{2-\alpha-\beta}} E_{\alpha, \alpha+\beta-1}\left[-(t-\tau)^{\alpha}\right] d \tau, \\
& J_{C}^{T F C E}(x, t)=\frac{1}{x} \int_{0}^{t} \frac{Q^{T F C E}(x, \tau)}{(t-\tau)^{1-\alpha}} E_{\alpha, \alpha}\left[-(t-\tau)^{\alpha}\right] d \tau
\end{aligned}
$$

where $E_{\alpha, \beta}(z)$ is the Mittag-Leffler function defined in (A.22). In addition, the fluxes, (4.35) and (4.38) can be given in terms of the distribution-like (4.42) via

$$
\begin{aligned}
J_{R L}^{T F D E}(x, t) & =\frac{1}{x^{0^{+}}} D_{t}^{1-\beta} Q^{T F D E}(x, t), \\
J_{C}^{T F D E}(x, t) & =\frac{1}{x} Q^{T F D E}(x, t) .
\end{aligned}
$$

\section{Concluding remarks}

In this work we addressed four Cattaneo-type models with time fractality based on Riemann-Liouville and Caputo single-order and distributedorder fractional derivatives. Many processes which transit from ballistic or superdiffusive behaviour in the short-time limit to subdiffusive behaviour, or even without transport $\left\langle x^{2}(t)\right\rangle \sim 1$, in the long-time limit are found to be captured by these Cattaneo-like equations. We summarise the MSD of the proposed models in Table 1

Conditional closed-form solutions for the proposed models were derived in terms of the Fox $H$-function. Alternative formulations for the Green's function of normal, time-fractional, time-fractional finite-speed diffusion were provided. For the generalised Cattaneo equation of type I with two fractional parameters, this modified Green's function can be viewed as a gauge for the subordination to the Gaussian process, where the nonnegativity of the modified Green's function is broken in the transition from 
Gaussian to non-Gaussian. It is worth mentioning that we have disregarded the space-fractality which submits a generic crossover from superballistic/subdiffusion to subdiffusion/superballistic. Such space-fractional kinetic models can be found in the double-order space-fractional diffusion equation in the natural/modified forms [82, 15], the focus of a separate future work.

\section{Appendix A. Mathematical preliminaries}

In this appendix we give a short brief for the mathematical preliminaries needed throughout the paper.

A.1. Fractional derivatives and transform techniques. We start with the left-sided Caputo (C) fractional derivative of order $\alpha \in(0,1]$, defined for any well-behaved function $f(t)$ by [66, 35]

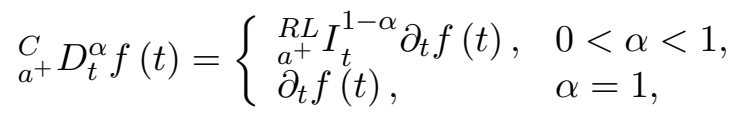

$\forall a \in \mathbb{R}$, where ${ }_{a^{+}}^{R L} I_{t}^{\alpha}$ is the left-sided Riemann-Liouville fractional integral given as

$$
{ }_{a^{+}}^{R L} I_{t}^{\alpha} f(t)= \begin{cases}\frac{1}{\Gamma(\alpha)} \int_{a}^{t} \frac{f(\tau)}{(t-\tau)^{1-\alpha}} d \tau, & \alpha>0, \\ f(t), & \alpha=0 .\end{cases}
$$

The left-sided Riemann-Liouville (RL) fractional derivative of order $\alpha \in$ $(0,1]$ is defined as

$$
{ }_{a^{+}}^{R L} D_{t}^{\alpha} f(t)= \begin{cases}\partial_{t}{ }_{a^{+}} I_{t}^{1-\alpha} f(t), & 0<\alpha<1, \\ \partial_{t} f(t), & \alpha=1 .\end{cases}
$$

The regularised Prabhakar fractional derivative is defined by [67, 22]

$$
{ }_{a^{+}}^{\mathcal{P}} D_{\alpha,-\lambda, t}^{\delta, \beta} f(t)=\int_{a}^{t}(t-\tau)^{n-1-\beta} E_{\alpha, n-\beta}^{-\delta}\left(-\lambda(t-\tau)^{\alpha}\right) \partial_{\tau}^{n} f(\tau) d \tau,
$$

where $n-1<\beta<n$, and $E_{\alpha, \beta}^{\delta}(z)$ is the Prabhakar generalisation of Mittag-Leffler function, see equation (A.16).

The Laplace transform $\mathcal{L}\{f(t) ; s\}=\int_{0}^{\infty} e^{-s t} f(t) d t=\tilde{f}(s)$ any generic function $f(t)$ is given for the $\mathrm{RL}$ and $\mathrm{C}$ fractional derivatives of $f(t)$ by

$\mathcal{L}\left\{\begin{array}{l}R L \\ 0^{+}\end{array} D_{t}^{\alpha} f(t) ; s\right\}=s^{\alpha} \tilde{f}(s), \quad \mathcal{L}\left\{\begin{array}{l}C \\ 0^{+}\end{array} D_{t}^{\alpha} f(t) ; s\right\}=s^{\alpha} \tilde{f}(s)-s^{\alpha-1} f\left(0^{+}\right)$, where $\alpha \in(0,1]$. The Fourier transform of any generic function $g(x)$ is defined by 


$$
\begin{aligned}
& \mathcal{F}\{g(x) ; k\}=\int_{-\infty}^{\infty} e^{-\imath k x} g(x) d x=\hat{g}(k), \\
& \mathcal{F}^{-1}\{\hat{g}(k) ; x\}=\frac{1}{2 \pi} \int_{-\infty}^{\infty} e^{\imath k x} \hat{g}(k) d x=g(x) .
\end{aligned}
$$

A.2. Fox $H$-function. The Fox $H$-function is defined in terms of the Mellin-Barnes integral 60]

$$
H_{p, q}^{m, n}\left[\begin{array}{l|l}
x & \begin{array}{c}
\left(a_{1}, A_{1}\right), \cdots,\left(a_{p}, A_{p}\right) \\
\left(b_{1}, B_{1}\right), \cdots,\left(b_{q}, B_{q}\right)
\end{array}
\end{array}\right]=\frac{1}{2 \pi \imath} \int_{\Omega} \Theta(s) x^{s} d s
$$

where $m, n, p$, and $q$ are integers satisfying $0 \leq n \leq p, 1 \leq m \leq q$, $a_{i}, b_{j} \in \mathbb{C}, A_{i}, B_{j} \in \mathbb{R}_{+}, i=1, \cdots, p, j=1, \cdots, q$, and the function $\Theta(s)$ is given by

$$
\Theta(s)=\frac{\prod_{j=1}^{m} \Gamma\left(b_{j}-B_{j} s\right) \prod_{j=1}^{n} \Gamma\left(1-a_{j}+A_{j} s\right)}{\prod_{j=m+1}^{q} \Gamma\left(1-b_{j}+B_{j} s\right) \prod_{j=n+1}^{p} \Gamma\left(a_{j}-A_{j} s\right)},
$$

where $\Gamma(\cdot)$ is the Gamma function. The contour $\Omega$ in the right side of equation (1) separates the poles of $\Gamma\left(b_{j}+B_{j} s\right), j=1, \cdots, m$ from the poles of $\Gamma\left(1-a_{i}-A_{i} s\right), i=1, \cdots, n$.

The Mellin transform, defined for any generic function as $\mathcal{M}\{f(x)\}(z)=$ $\int_{0}^{\infty} x^{z-1} f(x) d x$, of the $\mathrm{H}$-function is given by

$$
\int_{0}^{\infty} x^{z-1} H_{p, q}^{m, n}\left[a x \mid \begin{array}{c}
\left(a_{p}, A_{p}\right) \\
\left(b_{q}, B_{q}\right)
\end{array}\right] d x=a^{-z} \Theta(-z),
$$

where $\Theta(z)$ is given by (A.8). by

The inverse Fourier transform of the $H$-function $H_{1,2}^{1,1}\left[a|k|^{\delta}\right]$ is given

$$
\begin{aligned}
& \mathcal{F}^{-1}\left\{|k|^{\lambda} H_{1,2}^{1,1}\left[a|k|^{\delta} \mid \begin{array}{l}
(-n, 1) \\
(0,1) ;(\beta, \gamma)
\end{array}\right]\right\}(x) \\
& =\frac{1}{\sqrt{4 \pi} a^{\frac{\lambda+1}{\delta}}} H_{2,3}^{2,1}\left[\frac{|x|^{\delta}}{2^{\delta} a} \mid \begin{array}{l}
\left(1-\frac{\lambda+1}{\delta}, 1\right) ;\left(1-\beta-\frac{\lambda+1}{\delta} \gamma, \gamma\right) \\
\left(0, \frac{\delta}{2}\right),\left(1+n-\frac{\lambda+1}{\delta}, 1\right) ;\left(\frac{1}{2}, \frac{\delta}{2}\right)
\end{array}\right],
\end{aligned}
$$

where $k$ is the Fourier variable, $a, \gamma, \delta \in \mathbb{R}_{+}, \beta, n \in \mathbb{C}$, and $\lambda \in \mathbb{R}_{+} \cup\{0\}$.

REMARK A.1.The proof of relation (A.10) has been omitted for brevity. For more details about the method, the reader may consult [20, 56, 36].

If the poles of $\prod_{j=1}^{m} \Gamma\left(b_{j}-B_{j} s\right)$ are simple, the following series expansion holds true 


$$
\begin{aligned}
H_{p, q}^{m, n}\left[x \mid \begin{array}{c}
\left(a_{p}, A_{p}\right) \\
\left(b_{q}, B_{q}\right)
\end{array}\right]=\sum_{h=1}^{m} \sum_{\nu=0}^{\infty} \frac{(-1)^{\nu} x^{\frac{b_{h}+\nu}{B_{h}}}}{\nu ! B_{h}} \\
\times \frac{\prod_{j=1, j \neq h}^{m} \Gamma\left(b_{j}-B_{j} \frac{b_{h}+\nu}{B_{h}}\right) \prod_{j=1}^{n} \Gamma\left(1-a_{j}+A_{j} \frac{b_{h}+\nu}{B_{h}}\right)}{\prod_{j=m+1}^{q} \Gamma\left(1-b_{j}+B_{j} \frac{b_{h}+\nu}{B_{h}}\right) \prod_{j=n+1}^{p} \Gamma\left(a_{j}-A_{j} \frac{b_{h}+\nu}{B_{h}}\right)} .
\end{aligned}
$$

The following properties are used throughout the paper,

$$
\begin{aligned}
& H_{p, q}^{m, n}\left[x^{\delta} \mid \begin{array}{c}
\left(a_{p}, A_{p}\right) \\
\left(b_{q}, B_{q}\right)
\end{array}\right]=\frac{1}{\delta} H_{p, q}^{m, n}\left[x \mid \begin{array}{l}
\left(a_{p}, \frac{A_{p}}{\delta}\right) \\
\left(b_{q}, \frac{B_{q}}{\delta}\right)
\end{array}\right], \delta>0,
\end{aligned}
$$

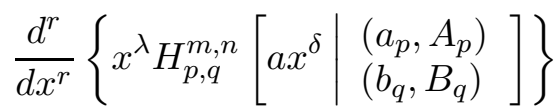

$$
\begin{aligned}
& =x^{\lambda-r} H_{p+1, q+1}^{m, n+1}\left[\begin{array}{l|l}
a x^{\delta} & \begin{array}{l}
(-\lambda, \delta)\left(a_{p}, A_{p}\right) \\
\left(b_{q}, B_{q}\right),(r-\lambda, \delta)
\end{array}
\end{array}\right],
\end{aligned}
$$

where $a, \delta \in \mathbb{R}_{+}$, and $r \in \mathbb{N} \cup\{0\}$,

$$
\begin{aligned}
& H_{p, q}^{m, n}\left[x \mid \begin{array}{l}
\left(a_{1}, A_{1}\right), \cdots,\left(a_{p}, A_{p}\right) \\
\left(b_{1}, B_{1}\right), \cdots,\left(b_{q-1}, B_{q-1}\right),\left(a_{1}, A_{1}\right)
\end{array}\right] \\
& =H_{p-1, q-1}^{m, n-1}\left[\begin{array}{l|l}
\left.x \mid \begin{array}{l}
\left(a_{2}, A_{2}\right), \cdots,\left(a_{p}, A_{p}\right) \\
\left(b_{1}, B_{1}\right), \cdots,\left(b_{q-1}, B_{q-1}\right)
\end{array}\right], n \geq 1, q>m,
\end{array}\right.
\end{aligned}
$$

and

$$
H_{0,1}^{1,0}[x \mid \overline{(b, B)}]=\frac{1}{B} x^{\frac{b}{B}} \exp \left(-x^{\frac{1}{B}}\right) .
$$

A.3. Generalised Mittag-Leffler functions. The Prabhakar generalisation of Mittag-Leffler function (PML) is defined in the series form [24]

$$
E_{\alpha, \beta}^{\gamma}(z)=\sum_{n=0}^{\infty} \frac{(\gamma)_{n}}{\Gamma(\alpha n+\beta)} \frac{z^{n}}{n !}, \quad \alpha, \beta, \gamma, z \in \mathbb{C}, \quad \operatorname{Re}\{\alpha\}>0,
$$

where $(\gamma)_{n}$ is the ascending Pochhammer symbol defined by $(\gamma)_{0}=1$, $(\gamma)_{n}=\gamma(\gamma+1) \cdots(\gamma+n-1)=\frac{\Gamma(\gamma+n)}{\Gamma(\gamma)}$. The PML function, $E_{\alpha, \beta}^{\gamma}\left(-\lambda t^{\alpha}\right)$ is a completely monotone function for $t \geq 0, \lambda$ is positive constant, $0<$ $\alpha, \beta \leq 1$, and $0<\gamma \leq \beta / \alpha$, has the asymptotic representation

$$
E_{\alpha, \beta}^{\gamma}\left(-\lambda t^{\alpha}\right) \sim \begin{cases}\frac{1}{\Gamma(\beta)}-\frac{\lambda \gamma t^{\alpha}}{\Gamma(\alpha+\beta)}, & t \rightarrow 0^{+} \\ \frac{\left(\lambda t^{\alpha}\right)-\gamma}{\Gamma(\beta-\alpha \gamma)}, & t \rightarrow \infty\end{cases}
$$


where the short-time behaviour is deduced from the series representation (A.16) and the long-time behaviour can be obtained from the series

$$
E_{\alpha, \beta}^{\gamma}(-z)=\frac{z^{-\gamma}}{\Gamma(\gamma)} \sum_{n=0}^{\infty} \frac{\Gamma(n+\gamma)}{\Gamma[\beta-\alpha(n+\gamma)]} \frac{(-z)^{-n}}{n !}, \quad|z|>1
$$

The most important properties of PML are its Laplace transform

$$
\mathcal{L}\left\{t^{\beta-1} E_{\alpha, \beta}^{\gamma}\left(-\lambda t^{\alpha}\right) ; s\right\}=\frac{s^{\alpha \gamma-\beta}}{\left(s^{\alpha}+\lambda\right)^{\gamma}},
$$

where $s$ is the Laplace variable, and its relation to the Fox $H$-function,

$$
E_{\alpha, \beta}^{\gamma}(-z)=\frac{1}{\Gamma(\gamma)} H_{1,2}^{1,1}\left[z \mid \begin{array}{l}
(1-\gamma, 1) \\
(0,1),(1-\beta, \alpha)
\end{array}\right] .
$$

The following recurrence relation can be validated by definition (A.16)

$$
E_{\alpha, \beta}^{\gamma+1}\left(-t^{\alpha}\right)+t^{\alpha} E_{\alpha, \beta+\alpha}^{\gamma+1}\left(-t^{\alpha}\right)=E_{\alpha, \beta}^{\gamma}\left(-t^{\alpha}\right) .
$$

When $\gamma=1$, the PML reduces to the generalised Mittag-Leffler function with two parameters,

$$
E_{\alpha, \beta}^{1}(z)=E_{\alpha, \beta}(z)=\sum_{n=0}^{\infty} \frac{z^{n}}{\Gamma(\alpha n+\beta)}, \quad \alpha, \beta, z \in \mathbb{C}, \quad \operatorname{Re}\{\alpha\}>0
$$

and when $\gamma=\beta=1$, the classical Mittag-Leffler function is recovered,

$$
E_{\alpha, 1}(z)=E_{\alpha}(z)=\sum_{n=0}^{\infty} \frac{z^{n}}{\Gamma(\alpha n+1)}, \quad \alpha, z \in \mathbb{C}, \quad \operatorname{Re}\{\alpha\}>0 .
$$

A.4. Unit doublet function. The unit doublet function (differentiator) [64] is defined as the first derivative of the Dirac delta function,

with the operational properties

$$
u_{1}(x)=\partial_{x} \delta(x)
$$

$$
\mathcal{F}\left\{u_{1}(x) ; k\right\}=\imath k, \quad \int_{-\infty}^{\infty} f(x-\xi) u_{1}(\xi) d \xi=\partial_{x} f(x) .
$$

It can be generalised to the $n$th differentiator function

$$
u_{n}(x)=\partial_{x}^{n} \delta(x)
$$

where $n \in \mathbb{N} \cup\{0\}$. When $n$ is negative integers, equation (A.26) defines then the $n$th integrator function, whereas $n=0$ recovers the conventional Dirac delta function (unit impulse function).

Let us introduce the "modified unit doublet" function as a product of the spatial variable $x$ and the unit doublet function,

whose Fourier transform is given by

$$
U_{1}(x)=x u_{1}(x),
$$

$$
\mathcal{F}\left\{U_{1}(x) ; k\right\}=-1=-\mathcal{F}\{\delta(x) ; k\} .
$$


Similar to the classical definition of the unit impulse function we consider the following forms for the unit doublet and its modification,

$$
\begin{aligned}
& \delta(x)=\lim _{a \rightarrow 0^{+}} \frac{1}{|a| \sqrt{\pi}} \exp \left(-\frac{x^{2}}{a^{2}}\right), \\
& u_{1}(x)=-\lim _{a \rightarrow 0^{+}} \frac{2 x}{a^{2}|a| \sqrt{\pi}} \exp \left(-\frac{x^{2}}{a^{2}}\right), \\
& U_{1}(x)=-\lim _{a \rightarrow 0^{+}} \frac{2 x^{2}}{a^{2}|a| \sqrt{\pi}} \exp \left(-\frac{x^{2}}{a^{2}}\right) .
\end{aligned}
$$

\section{A.5. Bernstein functions [8].}

Definition A.1. The function $f:(0, \infty) \rightarrow[0, \infty)$ is a completely monotone function if $f \in C^{\infty}$ and $f$ satisfies the Bernstein-HausdorffWidder condition; $(-1)^{n} f^{(n)}(t) \geq 0$ for all $n \in \mathbb{N}$. The family of all completely monotone functions is denoted by $\mathcal{C M F}$. The functions $\lambda^{\alpha}$ and $\exp (-a \lambda), a, \lambda>0, \alpha<0$, are typical examples of $\mathcal{C} \mathcal{M F}$. The product of two complete monotone functions and the linear combination of complete monotone functions are also a complete monotone function. The function $\varphi \in \mathcal{C} \mathcal{M F}$ if, and only if, there exists a function $f \geq 0$ such that $\varphi(\lambda)=\int_{0}^{\infty} \exp (-\lambda t) f(t) d t, \lambda>0$.

Definition A.2. The function $f:(0, \infty) \rightarrow[0, \infty)$ is a Bernstein function if $f \in C^{\infty}$ and $f$ satisfies the condition: $(-1)^{n-1} f^{(n)}(t) \geq 0$ for all $n \in \mathbb{N}$. The set of all Bernstein functions is denoted by $\mathcal{B F}$.

Definition A.3. The function $\varphi:(0, \infty) \rightarrow[0, \infty)$ is said to be a Stieltjes function if there exists $f \in \mathcal{C} \mathcal{M F}$ such that $\varphi(\lambda)=\int_{0}^{\infty} \exp (-\lambda t)$ $f(t) d t$, for $\lambda>0$. Denoting by $\mathcal{S F}$ to the family of all Stieltjes functions, it is obvious that $\mathcal{S F} \subset \mathcal{C} \mathcal{M F}$ since $f \geq 0$. The functions $\lambda^{-\alpha}, \lambda^{\alpha-1}$ and $\frac{1}{u+\lambda}$, where $\alpha \in[0,1], \lambda>0$ and $u>0$, are typical examples of Stieltjes function. The linear combination of Stieltjes functions are also a Stieltjes function.

Definition A.4. The Bernstein function $\varphi(\lambda)$ is said to be a complete Bernstein function if, and only if, $\varphi(\lambda) / \lambda \in \mathcal{S F}$. Denoting by $\mathcal{C B F}$ to the set of all complete Bernstein functions, thus $\mathcal{C B F} \subset \mathcal{B F}$. The functions $\lambda^{\alpha}$ and $\lambda^{1-\alpha}$, where $\alpha \in[0,1]$ and $\lambda>0$, are typical examples of complete Bernstein functions. The linear combination of complete Bernstein functions are also a complete Bernstein function. The set of complete Bernstein functions is not in general closed under multiplication, however, if $\varphi, \psi \in \mathcal{C B F}$, then $[\varphi(\lambda)]^{\alpha}[\psi(\lambda)]^{\beta} \in \mathcal{C B F}$ provided that $\alpha, \beta \in(0,1)$ and $\alpha+\beta \leq 1$. If $\varphi(\lambda) \in \mathcal{C B F}$, then $\exp (-a \varphi(\lambda)) \in \mathcal{C} \mathcal{M F}$ for $a, \lambda>0$. 


\section{Appendix B. Derivation of the solutions}

Here, we consider four different problems of inverting the LaplaceFourier transform, which are applied in the derivation of our solutions.

B.1. Problem B.1. Let us consider the inversion of the Laplace-Fourier transform of the following equation

$$
\widehat{\widetilde{\varrho}}(k, s)=\frac{s^{\gamma-1}+\chi_{0,1} s^{\beta-1}}{s^{\gamma}+\chi_{0,1} s^{\beta}+k^{2}},
$$

where $\chi_{0,1} \in\{0,1\}$ and $\gamma \geq \beta$ such that $\gamma=\beta$ if $\chi_{0,1}=0$ and $\gamma>\beta$ if $\chi_{0,1}=1$. Rearranging the above equation, we have

$$
\widehat{\widetilde{\varrho}}(k, s)=\frac{s^{\gamma-1}+\chi_{0,1} s^{\beta-1}}{s^{\gamma}+k^{2}}\left[1+f_{0}(k, s)\right]^{-1},
$$

where $f_{0}(k, s)=\frac{\chi_{0,1} s^{\beta}}{s^{\gamma}+k^{2}}$. When $\chi_{0,1}=1$, we note that $f_{0}(k, s)<1$ for $\gamma=$ $\alpha+\beta, 0<\alpha, \beta \leq 1, \operatorname{Re}\{s\}>0$, and $|k|>0.7$. These conditions provide a strong solution for Problem B.1. The long-space behaviours $(|k| \leq 0.7)$ are not of interest in our investigation. Using the condition that $f_{0}(k, s)<1$, then we have

$$
\widehat{\widetilde{\varrho}}(k, s)=\sum_{n=0}^{\infty}\left(-\chi_{0,1}\right)^{n}\left[\frac{s^{\beta n+\gamma-1}+\chi_{0,1} s^{\beta(n+1)-1}}{\left(s^{\gamma}+k^{2}\right)^{n+1}}\right],
$$

which can be converted to the physical domain by means of (A.10), A.19) and (A.20), and we obtain

$$
\begin{aligned}
& \varrho(x, t)=\sum_{n=0}^{\infty} \frac{\left(-\chi_{0,1}\right)^{n}}{n !} t^{(\gamma-\beta) n}\left[\varrho_{1}(x, t)+\chi_{0,1} t^{(\gamma-\beta)} \varrho_{2}(x, t)\right],
\end{aligned}
$$

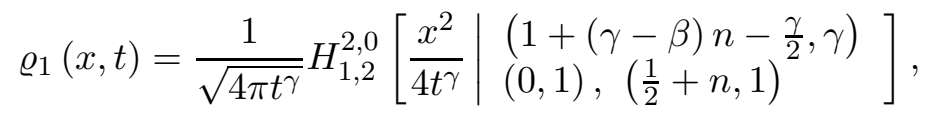

$$
\begin{aligned}
& \varrho_{2}(x, t)=\frac{1}{\sqrt{4 \pi t^{\gamma}}} H_{1,2}^{2,0}\left[\frac{x^{2}}{4 t^{\gamma}} \mid \begin{array}{l}
\left(1+(\gamma-\beta)(n+1)-\frac{\gamma}{2}, \gamma\right) \\
(0,1),\left(\frac{1}{2}+n, 1\right)
\end{array}\right] .
\end{aligned}
$$

REMARK B.1. When $\chi_{0,1}=0$ in (B.1), namely the only non-vanishing term is the first one $n=0, \sum_{n=0}^{\infty}\left(-\chi_{0,1}\right)^{n}=1$, and $\gamma=\beta$ then we have the classical solution of the time-fractional diffusion equation (TFDE) [48],

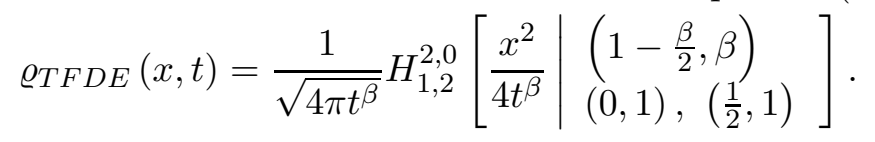

B.2. Problem B.2. If the well-behaved function $\varrho(x, t)$ has the LaplaceFourier transformed form

$$
\widehat{\widetilde{\varrho}}(k, s)=\frac{s^{\gamma-1}+\chi_{0,1} s^{\alpha-1}}{s^{\gamma}+\chi_{0,1} s^{\alpha}+k^{2}\left(p_{1}+p_{2} s^{\alpha-\beta}\right)},
$$


where $\chi_{0,1} \in\{0,1\}, \gamma \geq \alpha$ such that $\gamma=\alpha$ if $\chi_{0,1}=0$ and $\gamma>\alpha$ if $\chi_{0,1}=1$, $0<\alpha<\beta \leq 1$ and $p_{1}+p_{2}=1$, then

$$
\begin{aligned}
& \varrho(x, t)=\sum_{n=0}^{\infty} \frac{\left(-t^{\gamma-\alpha}\right)^{n}}{n !} \sum_{m=0}^{n}\left(\begin{array}{c}
n \\
m
\end{array}\right) \chi_{0,1}^{n-m}\left[\frac{p_{2}}{p_{1}} t^{\beta-\gamma}\right]^{m} \\
& \times\left[\varrho_{1}(x, t)+\chi_{0,1} t^{\gamma-\alpha} \varrho_{2}(x, t)\right], \\
& \varrho_{1}(x, t)=\frac{1}{\sqrt{4 \pi p_{1} t^{\gamma}}} \\
& \times H_{2,3}^{2,1}\left[\frac{x^{2}}{4 p_{1} t^{\gamma}} \mid \begin{array}{l}
\left.\left(\frac{1}{2}-m, 1\right) ;\left(1+(\gamma-\alpha) n+(\beta-\gamma) m-\frac{\gamma}{2}, \gamma\right)\right], \\
(0,1)+n-m, 1) ;\left(\frac{1}{2}, 1\right)
\end{array}\right] \\
& \varrho_{2}(x, t)=\frac{1}{\sqrt{4 \pi p_{1} t^{\gamma}}} \quad(0,1),\left(\frac{1}{2}+n-m, 1\right) ;\left(\frac{1}{2}, 1\right)
\end{aligned}
$$

provided that $p_{1} \geq p_{2}$. The proof of the above claim can be accomplished by firstly rewriting $\widehat{\varrho}(k, s)$ in the following form

$$
\widehat{\widetilde{\varrho}}(k, s)=\frac{s^{\gamma-1}+\chi_{0,1} s^{\alpha-1}}{s^{\gamma}+p_{1} k^{2}}\left[1+f_{1}\left(k, s ; p_{1}, p_{2}\right)\right]^{-1},
$$

where $f_{1}\left(k, s ; p_{1}, p_{2}\right)=\frac{\chi_{0,1} s^{\alpha}+p_{2} s^{\alpha-\beta} k^{2}}{s^{\gamma}+p_{1} k^{2}}$. By examining the properties of $f_{1}\left(k, \lambda ; p_{1}, p_{2}\right)$ for $\lambda=R e(s) \in \mathbb{R}_{+}$, we have the following cases: If $\chi_{0,1}=$ 0 and $\gamma=\alpha$, we have $f_{1}\left(k, \lambda ; p_{1}, p_{2}\right)=\frac{p_{2} \lambda^{\alpha-\beta} k^{2}}{\lambda^{\alpha}+p_{1} k^{2}}$ which satisfies that $f_{1}\left(k, \lambda ; p_{1}, p_{2}\right)<1$ for $\lambda>1,|k| \in \mathbb{R}$, provided that $p_{1} \geq p_{2}$. From these conditions, one can anticipate that the derived solution works well for the small and intermediate values of time, but it diverges for large values of time (for example $t=10$ ). Secondly, if $\chi_{0,1}=1$, and $\gamma=\alpha+1$, we have then $f_{1}\left(k, \lambda ; p_{1}, p_{2}\right)=\frac{\lambda^{\alpha}+p_{2} \lambda^{\alpha-\beta} k^{2}}{\lambda^{\alpha+1}+p_{1} k^{2}}$, which also satisfies that $f_{1}\left(k, \lambda ; p_{1}, p_{2}\right)<1$ for $\lambda>1,|k| \in \mathbb{R}$, provided that $p_{1} \geq p_{2}$. Following arguments not dissimilar to those in [77] and the solution of Problem B.1 lead us to (B.2).

REMARK B.2. If $\chi_{0,1}=0$ and $\gamma=\alpha$, then $\chi_{0,1}^{n-m}$ indicates that the only nonvanishing term of the second series of $(\underline{B .2})$ is the term $m=n$, namely,

$$
\begin{aligned}
\varrho(x, t)= & \frac{1}{\sqrt{4 \pi p_{1} t^{\alpha}}} \sum_{n=0}^{\infty} \frac{(-1)^{n}}{n !}\left[\frac{p_{2}}{p_{1}} t^{\beta-\alpha}\right]^{n} \\
& \times H_{2,3}^{2,1}\left[\frac{x^{2}}{4 p_{1} t^{\alpha}} \mid \begin{array}{c}
\left(\frac{1}{2}-n, 1\right) ;\left(1+(\beta-\alpha) n-\frac{\alpha}{2}, \alpha\right) \\
(0,1),\left(\frac{1}{2}, 1\right) ;\left(\frac{1}{2}, 1\right)
\end{array}\right],
\end{aligned}
$$


where $p_{1} \geq p_{2}$, which coincides the solution of the double-order timefractional diffusion equation of the modified form derived in [36].

B.3. Problem B.3. If the well-behaved function $\varrho(x, t)$ has the form

$$
\widehat{\widetilde{\varrho}}(k, s)=\frac{p_{1} s^{\gamma_{1}-1}+p_{2} s^{\gamma_{2}-1}+\chi_{0,1}\left(p_{1} s^{\alpha-1}+p_{2} s^{\beta-1}\right)}{p_{1} s^{\gamma_{1}}+p_{2} s^{\gamma_{2}}+\chi_{0,1}\left(p_{1} s^{\alpha}+p_{2} s^{\beta}\right)+k^{2}},
$$

in Laplace-Fourier space, where $0<\alpha<\beta \leq 1, p_{1}+p_{2}=1, \gamma_{1} \geq \alpha$ and $\gamma_{2} \geq \beta$ are given by

then

$$
\gamma_{1}=\left\{\begin{array}{ll}
\alpha, & \text { if } \chi_{0,1}=0, \\
\alpha+1, & \text { if } \chi_{0,1}=1,
\end{array} \quad \gamma_{2}= \begin{cases}\beta, & \text { if } \chi_{0,1}=0 \\
\beta+1, & \text { if } \chi_{0,1}=1\end{cases}\right.
$$

$$
\begin{aligned}
& \varrho(x, t)=\sum_{n=0}^{\infty} \frac{\left(-t^{\gamma_{2}-\gamma_{1}}\right)^{n}}{n !} \sum_{m=0}^{n}\left(\begin{array}{c}
n \\
m
\end{array}\right) \chi_{0,1}^{m}\left(\frac{p_{1}}{p_{2}}\right)^{n-m} \\
& \times t^{\left(\gamma_{1}-\beta\right) m} \sum_{\ell=0}^{m}\left(\begin{array}{c}
m \\
\ell
\end{array}\right)\left[\frac{p_{1}}{p_{2}} t^{\beta-\alpha}\right]^{\ell} \\
& \times\left\{\frac{p_{1}}{p_{2}} t^{\gamma_{2}-\gamma_{1}} \varrho_{1}(x, t)+\varrho_{2}(x, t)+\chi_{0,1} t^{\gamma_{2}-\beta}\left[\frac{p_{1}}{p_{2}} t^{\beta-\alpha} \varrho_{3}(x, t)+\varrho_{4}(x, t)\right]\right\}, \\
& \varrho_{1}(x, t)=\frac{1}{\sqrt{\frac{4 \pi t^{\gamma_{2}}}{p_{2}}}} \\
& \times H_{1,2}^{2,0}\left[\frac{p_{2} x^{2}}{4 t^{\gamma_{2}}} \mid \begin{array}{l}
\left(1+\gamma_{21}(n+1)+\left(\gamma_{1}-\beta\right) m+(\beta-\alpha) \ell-\frac{\gamma_{2}}{2}, \gamma_{2}\right) \\
(0,1),\left(\frac{1}{2}+n, 1\right)
\end{array}\right], \\
& \varrho_{2}(x, t)=\frac{1}{\sqrt{\frac{4 \pi t^{\gamma_{2}}}{p_{2}}}} \\
& \times H_{1,2}^{2,0}\left[\frac{p_{2} x^{2}}{4 t^{\gamma_{2}}} \mid \begin{array}{l}
\left(1+\gamma_{21} n+\left(\gamma_{1}-\beta\right) m+(\beta-\alpha) \ell-\frac{\gamma_{2}}{2}, \gamma_{2}\right) \\
(0,1),\left(\frac{1}{2}+n, 1\right)
\end{array}\right], \\
& \varrho_{3}(x, t)=\frac{1}{\sqrt{\frac{4 \pi t^{\gamma_{2}}}{p_{2}}}} \\
& \times H_{1,2}^{2,0}\left[\frac{p_{2} x^{2}}{4 t^{\gamma_{2}}} \mid \begin{array}{l}
\left(1+\gamma_{21}(n+1)+\left(\gamma_{1}-\beta\right) m+(\beta-\alpha) \ell+\gamma_{\alpha}-\frac{\gamma_{2}}{2}, \gamma_{2}\right) \\
(0,1),\left(\frac{1}{2}+n, 1\right)
\end{array}\right], \\
& \varrho_{4}(x, t)=\frac{1}{\sqrt{\frac{4 \pi t^{\gamma_{2}}}{p_{2}}}} \\
& \times H_{1,2}^{2,0}\left[\frac{p_{2} x^{2}}{4 t^{\gamma_{2}}} \mid \begin{array}{l}
\left(1+\gamma_{21} n+\left(\gamma_{1}-\beta\right) m+(\beta-\alpha) \ell+\gamma_{\beta}-\frac{\gamma_{2}}{2}, \gamma_{2}\right) \\
(0,1),\left(\frac{1}{2}+n, 1\right)
\end{array}\right],
\end{aligned}
$$


provided that $p_{1} \leq p_{2}$ for $\chi_{0,1}=0$, and $p_{1}<p_{2}$ for $\chi_{0,1}=1$. Here, we used $\gamma_{21}=\gamma_{2}-\gamma_{1}, \gamma_{\alpha}=\gamma_{1}-\alpha$, and $\gamma_{\beta}=\gamma_{2}-\beta$. form

In order to validate equation (B.3) we first rehash $\widehat{\widetilde{\varrho}}(k, s)$ to take the $\widehat{\widetilde{\varrho}}(k, s)=\frac{p_{1} s^{\gamma_{1}-1}+p_{2} s^{\gamma_{2}-1}+\chi_{0,1}\left(p_{1} s^{\alpha-1}+p_{2} s^{\beta-1}\right)}{p_{2} s^{\gamma_{2}}+k^{2}}\left[1+f_{2}\left(k, s ; p_{1}, p_{2}\right)\right]^{-1}$,

where

$$
f_{2}\left(k, s ; p_{1}, p_{2}\right)=\frac{p_{1} s^{\gamma_{1}}+\chi_{0,1}\left(p_{1} s^{\alpha}+p_{2} s^{\beta}\right)}{p_{2} s^{\gamma_{2}}+k^{2}} .
$$

Upon investigating the properties of $f_{2}\left(k, \lambda ; p_{1}, p_{2}\right), \lambda=\operatorname{Re}(s)>0$, we have two special cases of interest: When $\chi_{0,1}=0$, we get the characteristic function for DTFDE-II, given by $f_{2}\left(k, \lambda ; p_{1}, p_{2}\right)=\frac{p_{1} \lambda^{\alpha}}{p_{2} \lambda^{\beta}+k^{2}}$, with a graphical representation reports that for $p_{1} \leq p_{2}, \lambda>0,|k| \geq 0.6$ and $0<\alpha<\beta \leq 1$, then $f_{2}\left(k, \lambda ; p_{1}, p_{2}\right)<1$, otherwise, the resulting solution diverges. While, when $\chi_{0,1}=1$, we have $f_{2}\left(k, \lambda ; p_{1}, p_{2}\right)=\frac{p_{1} \lambda^{\alpha+1}+p_{1} \lambda^{\alpha}+p_{2} \lambda^{\beta}}{p_{2} \lambda^{\beta+1}+k^{2}}$, which satisfies $f_{2}\left(k, \lambda ; p_{1}, p_{2}\right)<1$ provided that $p_{1}<p_{2}, \lambda>0,|k| \geq 1$ and $0<\alpha<\beta \leq$ 1. We second apply the same method of proving Problem B.2, we arrive at (B.3).

REMARK B.3. The solution of the double-order time-fractional diffusion equation of the natural form obtained by 72 can be derived from (B.3) by setting $\chi_{0,1}=0$, and thus the only non-vanishing term of the second series is the first term, $m=0$, namely,

$$
\begin{gathered}
\varrho(x, t)=\sum_{n=0}^{\infty} \frac{(-1)^{n}}{n !}\left[\frac{p_{1}}{p_{2}} t^{\beta-\alpha}\right]^{n}\left\{\frac{p_{1}}{p_{2}} t^{\beta-\alpha} \varrho_{1}(x, t)+\varrho_{2}(x, t)\right\}, \\
\varrho_{1}(x, t)=\frac{1}{\sqrt{4 \pi t^{\beta} / p_{2}}} H_{1,2}^{2,0}\left[\frac{x^{2}}{4 t^{\beta} / p_{2}} \mid \begin{array}{c}
\left(1+(\beta-\alpha)(n+1)-\frac{\beta}{2}, \beta\right) \\
(0,1),\left(\frac{1}{2}+n, 1\right)
\end{array}\right], \\
\varrho_{1}(x, t)=\frac{1}{\sqrt{4 \pi t^{\beta} / p_{2}}} H_{1,2}^{2,0}\left[\frac{x^{2}}{4 t^{\beta} / p_{2}} \mid \begin{array}{c}
\left.1+(\beta-\alpha) n-\frac{\beta}{2}, \beta\right) \\
(0,1),\left(\frac{1}{2}+n, 1\right)
\end{array}\right],
\end{gathered}
$$

where $p_{1} \leq p_{2}$.

B.4. Problem B.4. If the well-behaved function $\varrho(x, t)$ is given by

$$
\widehat{\widetilde{\varrho}}(k, s)=\frac{\chi_{0,1}+p_{1} s^{\gamma_{1}-1}+p_{2} s^{\gamma_{2}-1}}{p_{1} s^{\gamma_{1}}+p_{2} s^{\gamma_{2}}+\chi_{0,1} s+k^{2}},
$$

in Laplace-Fourier space, where $p_{1}+p_{2}=1, \gamma_{1}$ and $\gamma_{2}$ are given through

$$
\gamma_{1}=\left\{\begin{array}{ll}
\alpha, & \text { if } \chi_{0,1}=0, \\
\alpha+1, & \text { if } \chi_{0,1}=1,
\end{array} \quad \gamma_{2}= \begin{cases}\beta, & \text { if } \chi_{0,1}=0, \\
\beta+1, & \text { if } \chi_{0,1}=1,\end{cases}\right.
$$


and $0<\alpha<\beta \leq 1$, then

$$
\begin{aligned}
& \varrho(x, t)=\sum_{n=0}^{\infty} \frac{(-1)^{n}}{n !}\left[\frac{t^{\gamma_{2}-1}}{p_{2}}\right]^{n} \sum_{m=0}^{n}\left(\begin{array}{c}
n \\
m
\end{array}\right) \chi_{0,1}^{n-m}\left[\frac{p_{1}}{t^{\gamma_{1}-1}}\right]^{m} \\
& \times\left\{\chi_{0,1} \frac{t^{\gamma_{2}-1}}{p_{2}} \varrho_{1}(x, t)+\frac{p_{1}}{p_{2}} t^{\gamma_{2}-\gamma_{1}} \varrho_{2}(x, t)+\varrho_{3}(x, t)\right\}, \\
& \varrho_{1}(x, t)=\frac{1}{\sqrt{4 \pi t^{\gamma_{2}} / p_{2}}} \\
& \times H_{1,2}^{2,0}\left[\frac{x^{2}}{4 t^{\gamma_{2}} / p_{2}} \mid \begin{array}{c}
\left(1+\left(\gamma_{2}-1\right)(n+1)-\left(\gamma_{1}-1\right) m-\frac{\gamma_{2}}{2}, \gamma_{2}\right) \\
(0,1),\left(\frac{1}{2}+n, 1\right)
\end{array}\right], \\
& \varrho_{2}(x, t)=\frac{1}{\sqrt{4 \pi t^{\gamma_{2}} / p_{2}}} \\
& \times H_{1,2}^{2,0}\left[\frac{x^{2}}{4 t^{\gamma_{2}} / p_{2}} \mid \begin{array}{c}
\left(1+\left(\gamma_{2}-1\right)(n+1)-\left(\gamma_{1}-1\right)(m+1)-\frac{\gamma_{2}}{2}, \gamma_{2}\right) \\
(0,1),\left(\frac{1}{2}+n, 1\right)
\end{array}\right], \\
& \varrho_{3}(x, t)=\frac{1}{\sqrt{4 \pi t^{\gamma_{2}} / p_{2}}} \\
& \times H_{1,2}^{2,0}\left[\frac{x^{2}}{4 t^{\gamma_{2}} / p_{2}} \mid \begin{array}{c}
\left(1+\left(\gamma_{2}-1\right) n-\left(\gamma_{1}-1\right) m-\frac{\gamma_{2}}{2}, \gamma_{2}\right) \\
(0,1),\left(\frac{1}{2}+n, 1\right)
\end{array}\right],
\end{aligned}
$$

provided that $p_{1} \leq p_{2}$ for $\chi_{0,1}=0$, and $p_{1} \in[0,0.3], p_{2} \in[0.7,1], p_{1}+p_{2}=1$, and $\beta \in[0.4,1]$ for $\chi_{0,1}=1$.

Remark B.4. We have written off the solution of Problem B.4. Setting $\chi_{0,1}=0$ in (B.4) results in again the solution of double-order time-fractional diffusion equation of the natural form [72].

\section{Acknowledgements}

RM acknowledges an Alexander von Humboldt Polish Honorary Research Scholarship from the Foundation for Polish Science (Fundacja na rzecz Nauki Polskiej).

\section{References}

[1] T.M. Atanackovic, S. Pilipovic, On a constitutive equation of heat conduction with fractional derivatives of complex order. Acta Mechanica 229, No 3 (2018), 1111-1121.

[2] E. Awad, On the time-fractional Cattaneo equation of distributed order. Physica A $\mathbf{5 1 8}$ (2019), 210-233.

[3] E. Awad, On the generalized thermal lagging behavior: Refined aspects. J. Thermal Stresses 35, No 4 (2012), 293-325. 
[4] E. Barkai, Y. Garini, R. Metzler, Strange kinetics of single molecules in living cells. Phys. Today 65, No 8 (2012), 29-35.

[5] E. Bazhlekova, Subordination in a class of generalized time-fractional diffusion-wave equations. Fract. Calc. Appl. Anal. 21, No 4 (2018), 869-900; DOI: 10.1515/fca-2018-0048; https://www.degruyter.com/view/j/fca.2018.21.issue-4/

issue-files/fca.2018.21.issue-4.xml.

[6] J.-P. Bouchaud, A. Georges, Anomalous diffusion in disordered media: statistical mechanisms, models and physical applications. Phys. Rep. 195, No 4-5 (1990), 127-293.

[7] J.-P. Bouchaud, A. Comtet, A. Georges, and P. Le Doussal, Classical diffusion of a particle in a one-dimensional random force field. Ann. Phys. (N.Y.) 201, No 2 (1990), 285-341.

[8] L. Boyadjiev, Y. Luchko, The neutral-fractional telegraph equation. Math. Modelling Nat. Phenomena 12, No 6 (2017), 51-67.

[9] M. Caputo, Distributed order differential equations modelling dielectric induction and diffusion. Fract. Calc. Appl. Anal. 4, No 4 (2001), 421442.

[10] C. Cattaneo, Sulla conduzione del calore. Atti Sem. Mat. Fis. Univ. Modena 3, No 3 (1948), 83-101.

[11] A. Chechkin, R. Gorenflo, I. Sokolov, Retarding subdiffusion and accelerating superdiffusion governed by distributed-order fractional diffusion equations. Phys. Rev. E 66, No 4 (2002), Art. 046129.

[12] A.V. Chechkin, J. Klafter, I.M. Sokolov, Fractional Fokker-Planck equation for ultraslow kinetics. Europhys. Lett. 63, No 3 (2003), 326332.

[13] A. Chechkin, V.Y. Gonchar, R. Gorenflo, N. Korabel, I. Sokolov, Generalized fractional diffusion equations for accelerating subdiffusion and truncated Lévy flights. Phys. Rev. E 78, No 2 (2008), Art. 021111.

[14] A.V. Chechkin, R. Gorenflo, I.M. Sokolov, V.Y. Gonchar, Distributed order time fractional diffusion equation. Fract. Calc. Appl. Anal. 6, No 3 (2003), 259-280.

[15] A. Chechkin, I.M. Sokolov, J. Klafter, Natural and modified forms of distributed-order fractional diffusion equations. In: Fractional Dynamics: Recent Advances, Ed. by J. Klafter, S.C. Lim, R. Metzler, World Scientific, Singapore (2011), 107-127.

[16] A. Compte, R. Metzler, The generalized Cattaneo equation for the description of anomalous transport processes. J. Phys. A 30, No 22 (1997), 7277-7289.

[17] A. Dhar, Fractional equation description of an open anomalous heat conduction set-up. J. Stat. Mech. 2019, No 1 (2019), Art. 013205. 
[18] A. Einstein, Über die von der molekularkinetischen Theorie der Wärme geforderte Bewegung von in ruhenden Flüssigkeiten suspendierten Teilchen. Ann. Phys. (Leipzig) 322, No 8 (1905), 549-560.

[19] A. Erdélyi, W. Magnus, F. Oberhettinger, F.G. Tricomi, Tables of Integral Transforms, Vol. 1. McGraw-Hill, New York (1954).

[20] W.G. Glöckle, T.F. Nonnenmacher, Fox function representation of non-Debye relaxation processes. J. Stat. Phys. 71, No 3-4 (1993), 741757.

[21] A. Godec, A. V. Chechkin, E. Barkai, H. Kantz, and R. Metzler, Localization and universal fluctuations in ultraslow diffusion processes. $J$. Phys. A 47, No 49 (2014), Art. 492002.

[22] R. Garra, R. Gorenflo, F. Polito, Ž. Tomovski, Hilfer-Prabhakar derivatives and some applications. Appl. Math. Comput. 242 (2014), 576589.

[23] R. Gorenflo, Y. Luchko, M. Stojanović, Fundamental solution of a distributed order time-fractional diffusion-wave equation as probability density. Fract. Calc. Appl. Anal. 16, No 2 (2013), 297-316; DOI: 10.2478/s13540-013-0019-6; https://www.degruyter.com/view/j/fca.2013.16.issue-2/ issue-files/fca.2013.16.issue-2.xml.

[24] R. Gorenflo, A.A. Kilbas, F. Mainardi, S.V. Rogosin, Mittag-Leffler Functions, Related Topics and Applications. Springer, Berlin (2014).

[25] R.A. Guyer, J.A. Krumhansl, Solution of the linearized phonon Boltzmann equation. Phys. Rev. 148, No 2 (1966), 766-778.

[26] S. Havlin and G. H. Weiss, A new class of long-tailed pausing time densities. J. Stat. Phys. 58, No 5-6 (1990), 1267-1273.

[27] R. Hilfer, Mathematical and physical interpretations of fractional derivatives and integrals. In: Handbook of Fractional Calculus with Applications, Vol. 1, Basic Theory, Ed. by A. Kochubei, Y. Luchko, Berlin, De Gruyter (2019).

[28] R. Hilfer, Fractional diffusion based on Riemann-Liouville fractional derivatives. J. Phys. Chem. B 104, No 16 (2000), 3914-3917.

[29] F. Höfling, T. Franosch, Anomalous transport in the crowded world of biological cells. Rep. Prog. Phys. 76, No 4 (2013), Art. 046602.

[30] P. De Jagher, A hyperbolic "diffusion equation" taking a finite collision frequency into account. Physica A 101, No 2-3 (1980), 629-633.

[31] J.-H. Jeon, H.M.-S. Monne, M. Javanainen, R. Metzler, Anomalous diffusion of phospholipids and cholesterols in a lipid bilayer and its origins. Phys. Rev. Lett. 109, No 18 (2012), Art. 188103.

[32] J.-H. Jeon, M. Javanainen, H. Martinez-Seara, R. Metzler, I. Vattulainen, Protein crowding in lipid bilayers gives rise to non-Gaussian 
anomalous lateral diffusion of phospholipids and proteins. Phys. Rev. $X$ 6, No 2 (2016), Art. 021006.

[33] D. Jou, G. Lebon, J. Casas-Vázquez, Extended Irreversible Thermodynamics. Springer, Berlin (2014).

[34] N.G. van Kampen, Stochastic Processes in Physics and Chemistry. North Holland, Amsterdam (1981).

[35] A.A. Kilbas, H.M. Srivastava, J.J. Trujillo, Theory and Applications of Fractional Differential Equations. Elsevier, Amsterdam (2006).

[36] T. Langlands, Solution of a modified fractional diffusion equation. Physica A 367 (2006), 136-144.

[37] P. Langevin, Sur la théorie du mouvement brownien. C. R. Acad. Sci. Paris 146, No 10 (1908), 530-533.

[38] Y.B. Lev, D.M. Kennes, C. Klöckner, D.R. Reichman, C. Karrasch, Transport in quasiperiodic interacting systems: From superdiffusion to subdiffusion. Europhys. Lett. 119, No 3 (2017), Art. 37003.

[39] Y. Luchko, Initial-boundary-value problems for the generalized multiterm time-fractional diffusion equation. J. Math. Anal. Appl. 374, No 2 (2011), 538-548.

[40] F. Mainardi, Fractional relaxation-oscillation and fractional diffusionwave phenomena. Chaos, Solitons \& Fractals 7, No 9 (1996), 14611477.

[41] F. Mainardi, The fundamental solutions for the fractional diffusionwave equation. Appl. Math. Lett. 9, No 6 (1996), 23-28.

[42] R. Gorenflo, F. Mainardi, Fractional calculus. In: Fractals and Fractional Calculus in Continuum Mechanics, Springer, Berlin (1997), 223276.

[43] F. Mainardi, G. Pagnini, R. Gorenflo, Some aspects of fractional diffusion equations of single and distributed order. Applied Mathematics and Computation 187, No 1 (2007), 295-305.

[44] R.N. Mantegna, H.E. Stanley, Stochastic process with ultraslow convergence to a Gaussian: the truncated Lévy flight. Phys. Rev. Lett. 73, No 22 (1994), 2946-2949.

[45] J. Masoliver, Fractional telegrapher's equation from fractional persistent random walks. Phys. Rev. E 93, No 5 (2016), Art. 052107.

[46] J. Masoliver, G.H. Weiss, Finite-velocity diffusion. Euro. J. Phys. 17, No 4 (1996), 190-196.

[47] Y. Meroz, I.M. Sokolov, A toolbox for determining subdiffusive mechanisms. Phys. Rep. 573 (2015), 1-29.

[48] R. Metzler, J. Klafter, The random walk's guide to anomalous diffusion: a fractional dynamics approach. Phys. Rep. 339, No 1 (2000), $1-77$. 
CROSSOVER DYNAMICS FROM SUPERDIFFUSION TO ... 99

[49] R. Metzler, J. Klafter, The restaurant at the end of the random walk: recent developments in the description of anomalous transport by fractional dynamics. J. Phys. A 37, No 31 (2004), R161-R208.

[50] R. Metzler, J. Klafter, I.M. Sokolov, Anomalous transport in external fields: Continuous time random walks and fractional diffusion equations extended. Phys. Rev. E 58, No 2 (1998), 1621-1633.

[51] R. Metzler, Generalized Chapman-Kolmogorov equation: A unifying approach to the description of anomalous transport in external fields. Phys. Rev. E 62, No 5 (2000), 6233-6245.

[52] R. Metzler, E. Barkai, and J. Klafter, Anomalous diffusion and relaxation close to thermal equilibrium: A fractional Fokker-Planck equation approach. Phys. Rev. Lett. 82, No 18 (1999), 3563-3567.

[53] R. Metzler, E. Barkai, and J. Klafter, Deriving fractional FokkerPlanck equations from a generalised master equation. Europhys. Lett. 46, No 4 (1999), 431-436.

[54] R. Metzler, J.-H. Jeon, and A. G. Cherstvy, Non-Brownian diffusion in lipid membranes: experiments and simulations. Biochimica et Biophysica Acta (BBA) - Biomembranes 1858, No 10 (2016), 2451-2467.

[55] R. Metzler, J.-H. Jeon, A.G. Cherstvy, E. Barkai, Anomalous diffusion models and their properties: non-stationarity, non-ergodicity, and ageing at the centenary of single particle tracking. Phys. Chem. Chem. Phys. 16 (2014), 24128-24164.

[56] R. Metzler, T.F. Nonnenmacher, Space- and time-fractional diffusion and wave equations, fractional Fokker-Planck equations, and physical motivation. Chem. Phys. 284, No 1-2 (2002), 67-90.

[57] D. Molina-Garcia, T. Sandev, H. Safdari, G. Pagnini, A. Chechkin, R. Metzler, Crossover from anomalous to normal diffusion: truncated power-law noise correlations and applications to dynamics in lipid bilayers. New J. Phys. 20 (2018), Art. 103027.

[58] P.M. Morse, H. Feshbach, Methods of Theoretical Physics. McGrawHill, New York (1953).

[59] F. Mainardi, A. Mura, G. Pagnini, R. Gorenflo, Time-fractional diffusion of distributed order. J. Vibration and Control 14, No 9-10 (2008), $1267-1290$.

[60] A.M. Mathai, R.K. Saxena, H.J. Haubold, The H-Function: Theory and Applications. Springer, Berlin (2010).

[61] R.R. Nigmatullin, The realization of the generalized transfer equation in a medium with fractal geometry. Phys. Status Solidi 133, No 1 (1986), 425-430. 
[62] T. Nonnenmacher, D. Nonnenmacher, Towards the formulation of a nonlinear fractional extended irreversible thermodynamics. Acta Phys. Hungarica 66, No 1-4 (1989), 145-154.

[63] K. Nørregaard, R. Metzler, C. Ritter, K. Berg-Sørensen, L.B. Oddershede, Manipulation and motion of organelles and single molecules in living cells. Chem. Rev. 117, No 5 (2017), 4342-4375.

[64] A.V. Oppenheim, Signals and Systems, MIT OpenCourseWare, Cambridge MA (2011); https://ocw.mit.edu/resources/

res-6-007-signals-and-systems-spring-2011.

[65] E. Orsingher, L. Beghin, Time-fractional telegraph equations and telegraph processes with Brownian time. Probability Theory and Related Fields 128, No 1 (2004), 141-160.

[66] I. Podlubny, Fractional Differential Equations: An Introduction to Fractional Derivatives, Fractional Differential Equations, to Methods of Their Solution and Some of Their Applications. Elsevier, Amsterdam (1998).

[67] T.R. Prabhakar, A singular integral equation with a generalized Mittag Leffler function in the kernel. Yokohama Math. J. 19, No 1 (1971), 7-15.

[68] L.F. Richardson, Atmospheric diffusion shown on a distance-neighbour graph. Proc. Roy. Soc. A 110, No 756 (1926), 709-737.

[69] H. Qi, X. Jiang, Solutions of the space-time fractional Cattaneo diffusion equation. Physica A 390, No 11 (2011), 1876-1883.

[70] T.Q. Qiu, C.L. Tien, Short-pulse laser heating on metals. Intl. J. Heat and Mass Transfer 35, No 3 (1992), 719-726.

[71] T.Q. Qiu, C.L. Tien, Heat transfer mechanisms during short-pulse laser heating of metals. ASME J. Heat Transfer 115, No 4 (1993), 835-841.

[72] T. Sandev, A.V. Chechkin, N. Korabel, H. Kantz, I.M. Sokolov, R. Metzler, Distributed-order diffusion equations and multifractality: Models and solutions. Phys. Rev. E 92, No 4 (2015), Art. 042117.

[73] T. Sandev, A. Chechkin, H. Kantz, R. Metzler, Diffusion and FokkerPlanck-Smoluchowski equations with generalized memory kernel. Fract. Calc. Appl. Anal. 18, No 4 (2015), 1006-1038; DOI: 10.1515/fca-20150059; https://www.degruyter.com/view/j/fca.2015.18.issue-4/ issue-files/fca.2015.18.issue-4.xml.

[74] T. Sandev, I.M. Sokolov, R. Metzler, A. Chechkin, Beyond monofractional kinetics. Chaos, Solitons \& Fractals 102 (2017), 210-217.

[75] T. Sandev, Z. Tomovski, J.L. Dubbeldam, A. Chechkin, Generalized diffusion-wave equation with memory kernel. J. Phys. A 52, No 1 (2019), Art. 015201. 
CROSSOVER DYNAMICS FROM SUPERDIFFUSION TO ... 101

[76] C. Sándor, A. Libál, C. Reichhardt, C. Olson Reichhardt, Dewetting and spreading transitions for active matter on random pinning substrates. J. Chem. Phys. 146, No 20 (2017), Art. 204903.

[77] R.K. Saxena, G. Pagnini, Exact solutions of triple-order time-fractional differential equations for anomalous relaxation and diffusion I: The accelerating case. Physica A 390, No 4 (2011), 602-613.

[78] R.L. Schilling, R. Song, Z. Vondracek, Bernstein Functions: Theory and Applications. De Gruyter, Berlin (2012).

[79] W. Schneider, W. Wyss, Fractional diffusion and wave equations. J. Math. Phys. 30, No 1 (1989), 134-144.

[80] Y.G. Sinai, The limiting behavior of a one-dimensional random walk in a random medium. Theory of Probability \& Applications 27, No 2 (1982), 256-268.

[81] M. von Smoluchowski, Zur kinetischen Theorie der Brownschen Molekularbewegung und der Suspensionen. Ann. Phys. (Leipzig) 326, No 14 (1906), 756-780.

[82] I. Sokolov, A. Chechkin, J. Klafter, Distributed-order fractional kinetics. Acta Phys. Polonica B 35, No 47 (2004), 1323-1341.

[83] I. Sokolov, Thermodynamics and fractional Fokker-Planck equations. Phys. Rev. E 63, No 5 (2001), Art. 056111.

[84] P. Tan, Y. Liang, Q. Xu, E. Mamontov, J. Li, X. Xing, L. Hong, Gradual crossover from subdiffusion to normal diffusion: a many-body effect in protein surface water. Phys. Rev. Lett. 120, No 24 (2018), Art. 248101.

[85] A.M. Tawfik, H. Fichtner, R. Schlickeiser, A. Elhanbaly, Analytical solutions of the space-time fractional telegraph and advection-diffusion equations. Physica A 491 (2018), 810-819.

[86] D.Y. Tzou, Macro-to Microscale Heat Transfer: The Lagging Behavior. John Wiley \& Sons, New York (2014).

[87] G.H. Weiss, Some applications of persistent random walks and the telegrapher's equation. Physica A 311, No 3-4 (2002), 381-410.

[88] W. Wyss, The fractional diffusion equation. J. Math. Phys. 27, No 11 (1986), 2782-2785.

[89] M.A. Zaks, A. Nepomnyashchy, Subdiffusive and superdiffusive transport in plane steady viscous flows. Proc. Natl. Acad. Sci. USA 116, No 37 (2018), 18245-18250.

[90] G.M. Zaslavsky, Hamiltonian Chaos and Fractional Dynamics. Oxford University Press, Oxford (2008).

[91] V. Želi, D. Zorica, Analytical and numerical treatment of the heat conduction equation obtained via time-fractional distributed-order heat conduction law. Physica A 492 (2018), 2316-2335. 
${ }^{1}$ Department of Mathematics, Faculty of Education Alexandria University

Souter St., El-Shatby, P.O. Box 21526

Alexandria, EGYPT

e-mail: emadawad78@alexu.edu.eg

${ }^{2}$ Institute of Physics \& Astronomy

University of Potsdam

Karl-Liebknecht-Str. 24/25

D-14476 Potsdam-Golm, GERMANY

e-mail: rmetzler@uni-potsdam.de

Received: August 7, 2019

Please cite to this paper as published in:

Fract. Calc. Appl. Anal., Vol. 23, No 1 (2020), pp. 55-102,

DOI: $10.1515 /$ fca-2020-0003 